\title{
PALINOLOGÍA DE LA FORMACIÓN ANDAPAICO (CISURALIANO-GUADALUPIANO), PRECORDILLERA CENTRAL SANJUANINA (ARGENTINA): CONSIDERACIONES BIOESTRATIGRÁFICAS REGIONALES
}

\author{
MARÍA LUCÍA BALARINO \\ Sección Paleopalinología, Museo Argentino de Ciencias Naturales "Bernardino Rivadavia", CONICET, \\ Av. Ángel Gallardo 470, C1405DJR, Buenos Aires, Argentina. lubalarino@macn.gov.ar
}

GUSTAVO A. CORREA

Área Paleontología, Instituto y Museo de Ciencias Naturales, CONICET, Av. España, 400, norte, San Juan, Argentina. gustavoalejandrocorrea@yahoo.com.br

PEDRO RAUL GUTIÉRREZ \& MARÍA L. CARREVEDO

Sección Paleopalinología, Museo Argentino de Ciencias Naturales "Bernardino Rivadavia", CONICET, Av. Ángel Gallardo,470,C1405DJR, Buenos Aires,Argentina.prgutierrez@macn.gov.ar,mlcarrevedo@macn.gov.ar

\begin{abstract}
PALYNOLOGY OF THE ANDAPAICO FORMATION (CISURALIAN-GUADALUPIAN), PRECORDILLERA OF CENTRAL SANJUANINA (ARGENTINA): REGIONAL BIOSTRATIGRAPHICAL CONSIDERATIONS. Presented here is a palynological study of samples from the upper part of the Andapaico Formation, Precordillera of San Juan, Argentina. The samples are located just above the eolian facies and have been referred to the Lueckisporites-Weylandites Biozone (LW), probably the basal part, based on comparison with other palynofloras described for the Central-West basins of Argentina. The association is early Cisuralian-middle Guadalupian in age. In addition, 40 species are proposed to be included as new elements unique to the LW Biozone. Comparisons with coeval palynofloras of the Chacoparaná (Argentina), Paraná, and Amazonas (Brazil) biozones were made, showing a high degree of correspondence with the Striatites, Lueckisporites costabilis-Vittatina virkkiae and Vittatina costabilis biozones respectively. According to the botanical affinities of the elements identified in the association, it is possible to infer that they represent a mesophilic flora, with a low participation of hygro-hydrophilic elements, reflecting the absence of wet conditions in areas close to the site of deposition.
\end{abstract}

Key words: Palynology, Permian, Andapaico Formation, San Juan, Argentina.

RESUMO - Apresenta-se aqui o estudo palinológico de amostras provenientes do topo do Formação Andapaico, Precordillera de San Juan, Argentina. Essas amostras estão localizadas logo acima das fácies eólicas e têm sido atribuídas à Biozona (LW) Lueckisporites-Weylandites, muito provavelmente a sua parte inferior, a partir da comparação com outras palinofloras descritas para as bacias do Centro-Oeste da Argentina. A idade da associação analisada é Cisuraliano inferior-Guadalpiano médio. Propõe-se também incluir 40 espécies como novos elementos exclusivos para a biozona LW. Esta palinoflora é parcialmente equivalente às biozonas Striatites (bacia Chacoparaná, Argentina), Lueckisporites costabilis - Vittatina virkkiae (Lc - Vv, da bacia do Paraná, Brasil) e Vittatina costabilis (Vc, da bacia do Amazonas, Brasil). De acordo com as possíveis afinidades botânicas dos elementos identificados na associação, é possível inferir que eles representam uma flora mesofílica, com baixa participação de elementos higro-hidrofílicos, refletindo a ausência de condições chuvosas propícias ao seu desenvolvimento nas áreas próximas à área de deposição.

Palavras-chave: Palinologia, Permiano, Formação Andapaico, San Juan, Argentina.

\section{INTRODUCCIÓN}

El registro estratigráfico del margen occidental del Gondwana en el Paleozoico tardío está documentado por diferentes avances y retrocesos del mar (Limarino \& Spalletti, 2006), evidenciado por el desarrollo de una serie de cuencas orientales y occidentales en el extremo sur de Sudamérica. Una de las cuencas occidentales es la Cuenca Paganzo (Figura 1), que habría estado limitada al oeste por la Protoprecordillera (Salfity \& Gorustovich, 1983; González Bonorino, 1991), y comunicada a través de ésta barrera con la Cuenca CalingastaUspallata, predominantemente marina (Frakes, 1979). En el área de desarrollo de la Cuenca Paganzo se han registrado estas ingresiones que aparecen como sucesiones silicoclásticas en afloramientos casi continuos entre el sur de Jáchal (Provincia de San Juan) y el norte de la Provincia de Mendoza 


\section{Referencias}

\section{Dv Formación Punta Negra $\quad$ Elgas éreas emergidas \\ Pm Formación Andapaico $\quad 1$ Afloramiento en estudio \\ Tc Formación Albarracín 2 Protoprecordillera}
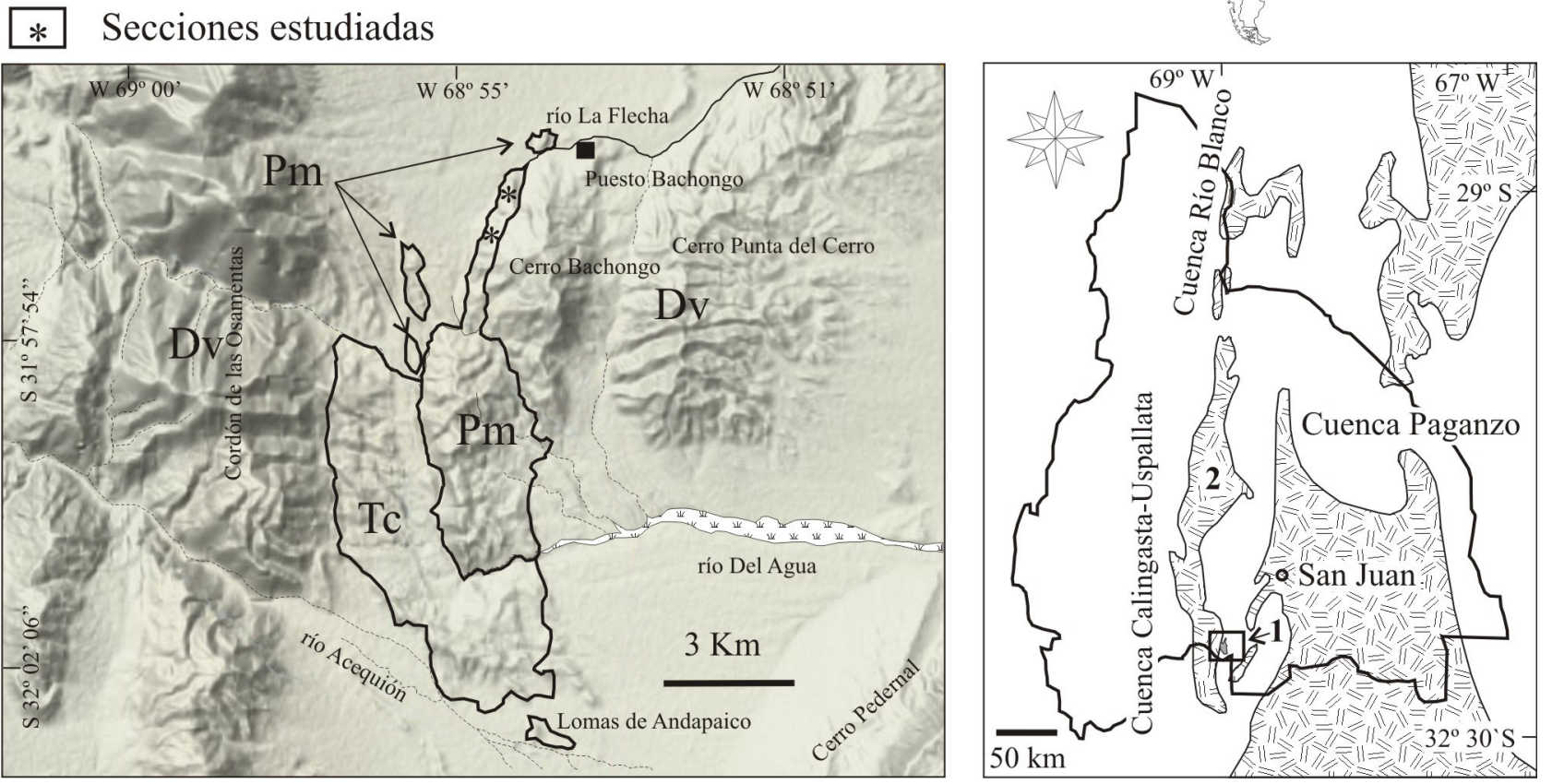

Figura 1. Ubicación geográfico de los afloramientos de la Formación Andapaico (modificado de Salfity \& Gorustovich, 1983).

Figure 1. Geographic location of the Andapaico Formation (modified from Salfity \& Gorustovich, 1983).

que forman fajas alargadas en sentido N-S (Limarino et al., 1996; Ramos \& Vujovich, 2000). Dichos afloramientos recibieron diferentes nombres formacionales (i.e. Guandacol-Tupe-Patquía; Río Francia-Patquía; La Deheza-Ojo de Agua y Andapaico) según el área donde fueron caracterizadas (véase Azcuy et al., 2000). Los análisis sedimentológicos y paleontológicos realizados en estas sucesiones aportaron información novedosa para su interpretación paleoambiental y cronológica (Correa et al., 2012; Gutiérrez et al., 2010, 2011), ayudando a completar el esquema evolutivo de la sedimentación en la Cuenca Paganzo (Limarino et al., 2002, 2006).

En esta contribución se presenta un estudio palinológico de la sección superior de la Formación Andapaico, el cual permite acotar la edad del techo de la unidad y aporta nuevos elementos de correlación para las floras desarrolladas durante el Pérmico Inferior-Medio en los sectores más australes de la Cuenca Paganzo.

\section{MATERIAL Y MÉTODOS}

El material estudiado proviene de la parte superior de la Formación Andapaico, Precordillera Central de la Provincia de San Juan, Argentina. En el perfil estudiado de identificaron siete asociaciones de facies (Figura 2), siendo la séptima la portadora de tres niveles microflorísticos analizados: PBSJ 374,362 y 361 . Se estudiaron 21 preparados del nivel 374, diez del nivel 362 y 6 del nivel 361. Para su procesamiento se han seguido las técnicas convencionales para muestras palinológicas (Wood et al., 1996). Los estudios sistemáticos y las fotografías tomadas se centraron en el nivel microflorístico 374 , por presentar el mejor grado de preservación de los ejemplares. Los preparados microscópicos se hallan depositados en la Colección del Instituto y Museo de Ciencias Naturales, Universidad Nacional de San Juan, bajo la sigla PBSJ. Las coordenadas del material ilustrado son brindadas según la reglilla England Finder. Las fotografías fueron tomadas con una máquina fotográfica digital marca Nikon DSFil, adosada a un microscopio Nikon modelo Eclipse 50i. Para ampliar estudios morfológicos se montaron muestras para microscopio electrónico de barrido (MEB) marca Philips serie XL modelo 30, perteneciente al Museo Argentino de Ciencias Naturales. Para la clasificación sistemática supragenérica de las esporas se siguió el esquema de Potonié \& Kremp (1954) y modificaciones posteriores (sintetizado en Playford \& Dettmann, 1996). Las citas bibliográficas que son incluidas en la bibliografía son referidas a partir de la categoría Género.

\section{GEOLOGÍA DEL ÁREA}

La Formación Andapaico aflora en la región sur de la 


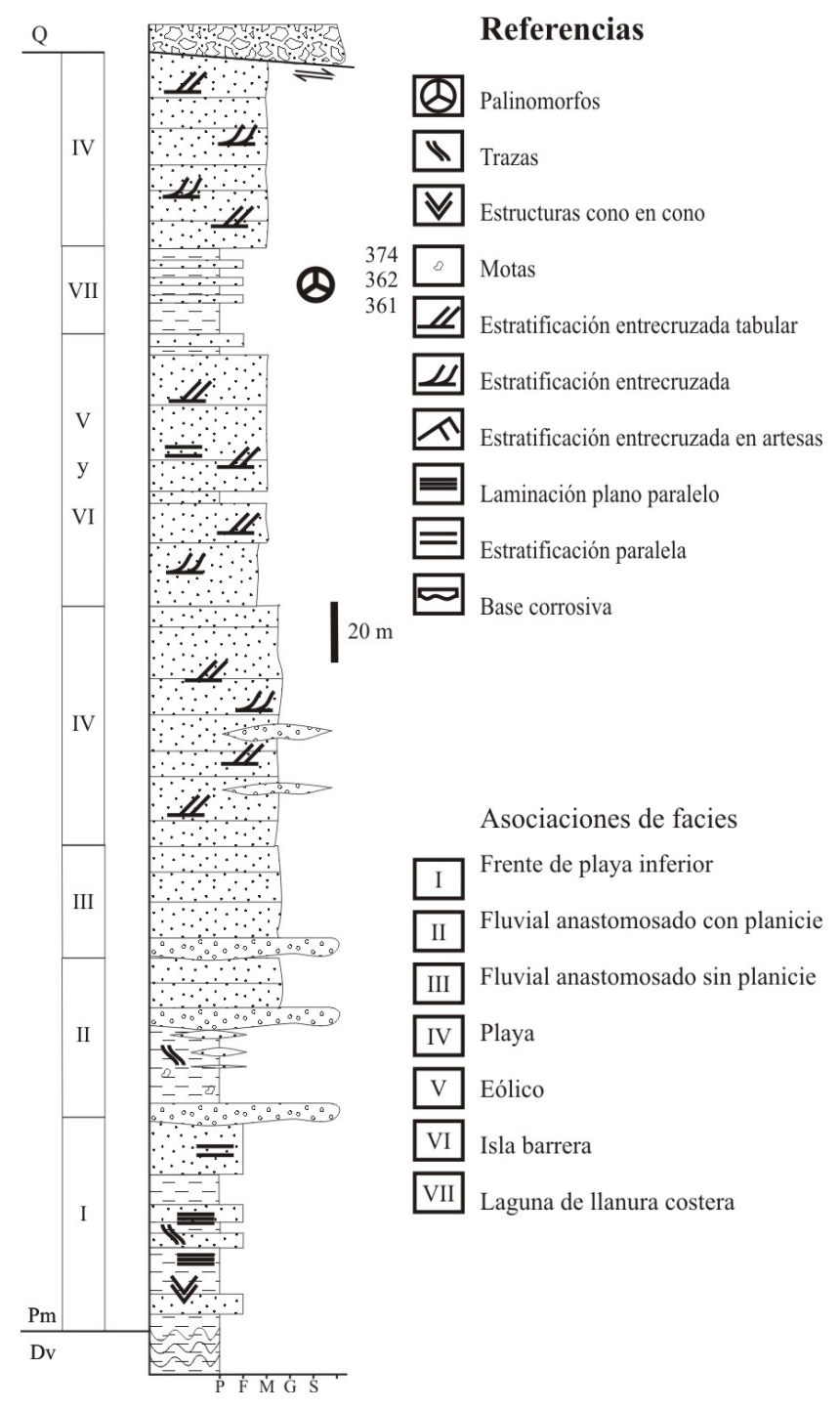

Figura 2. Perfil esquemático de la Formación Andapaico, con las ubicaciones de las muestras palinológicas y las facies sedimentarias.

Figure 2. Stratigraphical section of the Andapaico Formation, showing sampling level, lithology and sedimentary facies.

Provincia de San Juan, entre el Puesto Bachongo (al norte) y Lomas de Andapaico (al sur) (Figura 1), constituyendo una faja elongada en sentido N-S de $18 \mathrm{~km}$ de largo y 1,5 $\mathrm{km}$ de ancho máximo. Se apoya en discordancia angular sobre lutitas y psamitas devónicas de la Formación Punta Negra (Bracaccini, 1950), mientras que el techo está biselado por una falla N-S, que la pone en contacto con depósitos de abanicos aluviales cuaternarios. Esta unidad, definida por Harrington (1971) y referida originalmente al Pennsylvaniano, fue poco estudiada en especial en cuanto sus aspectos sedimentológicos y paleoambientales (Arrondo et al., 1986, 1990; Coca \& Bercowski, 1994; Olivares Milla, 2002). En cuanto a su contenido paleontológico, se destaca el hallazgo de Arrondo et al. (1986), quienes describieron restos megaflorísticos de tres niveles (ubicados a 37, 47 y $253 \mathrm{~m}$ de la base de la unidad) y localizados en las proximidades del Puesto Bachongo (Figura 1). Esta flora fue asignada a la Biozona de Intervalo (e.g. Archangelsky \& Arrondo en Archangelsky, 1971; Archangelsky \& Azcuy, 1985; Arrondo et al., 1986; Archangelsky \& Cúneo, 1991) que caracteriza al Pennsylvaniano Superior del oeste argentino.

Posteriormente, Correa et al. (2012) dieron a conocer novedosos hallazgos micro y megaflorísticos que permiten reubicar la Formación Andapaico en el intervalo Cisuraliano temprano-Guadalupiano, modificando la edad aceptada para la unidad (Pennsylvaniano Tardío-Pérmico; Harrington, 1971; Arrondo et al., 1986; Gutiérrez et al., 1992; Coca \& Bercowski, 1994).

En esta contribución se ilustra y describe las asociaciones palinológicas de la parte superior de la Formación Andapaico (PBSJ 374, 362, 361), cuyos primeros datos fueran adelantados en forma provisoria por Correa et al. (2012).

Los niveles portadores de las microfloras aquí analizadas fueron colectados entre el Puesto Bachongo y los $31^{\circ} 57^{\prime} \mathrm{S}$ (Figura 1). Allí, la sucesión, incluye $430 \mathrm{~m}$ de estratos homoclinales (de rumbos meridionales y buzamientos de $50^{\circ}$ al oeste), que fueron caracterizados como integrados por siete asociaciones de facies (Correa et al., 2012). La parte cuspidal de la sucesión está integrada por unos 94 $\mathrm{m}$ de depósitos pelíticos grises y rojos y areniscas medias macizas, estratificada planar y entrecruzada tabular planar (Figura 2) en las cuales se alternan depósitos costeros de asociaciones de facies de playa, islas barrera, eólicas y lagoon (Correa et al., 2012). Las pelitas grises de lagoon brindaron microfloras (PBSJ 374, 362 y 361), que son presentadas en este trabajo.

\section{RESULTADOS}

\section{Contenido palinológico}

La microflora analizada incluyendo ochenta y cuatro especies (siendo doce referibles a esporas trilete, diecinueve a granos de polen monosacado, diecinueve a bisacado liso, treinta y uno a bisacado teniado, dos a sulcado y un alga) son listados a continuación. Diez de estas especies mantienen su asignación taxonómica abierta a partir de los escasos ejemplares obtenidos de las mismas y su regular preservación, algunas de ellas son descriptas más abajo. La distribución de las especies identificadas en los tres niveles fosilíferos se presenta en la Tabla 1. La composición cuantitativa de cada uno de los niveles por su parte se presenta en las Tablas 2 y 3 . En ellos puede verse que las microfloras aparecen integradas por granos de polen teniado (28,5-49,5\%), granos monosacados $(11,3-36,4 \%)$, granos bisacados (17-22\%) y esporas trilete $(10,4-36,5 \%)$. A nivel de género es conspicua la presencia de Punctatisporites (1,8-15,5\%), Vittatina (5,1-13,1\%), Protohaploxypinus (4,3-7,9\%), Alisporites (4-7,2\%), Hamiapollenites (2-6,2\%), Vallatisporites (0,3-5,6\%), Mabuitasaccites (2,5-4,8\%), Scheuringipollenites $(0,6-4,4 \%)$. 
DESCRIPCIONES SISTEMÁTICAS

Anteturma VARIEGERMINANTES Potonié, 1970

Turma SACCITES Erdtman, 1947

Subturma MONOSACCITES Chitaley emend.

Potonié \& Kremp, 1954

Infraturma ALETESACCITES Leschik, 1955
Accinctisporites Leschik, 1955

Especie tipo. Accinctisporites ligatus Leschik, 1955.

Accinctisporites ligatus Leschik, 1955

in Kräusel \& Leschik, 1955

(Figuras 3I-J)

Tabla 1. Distribución de las especies identificadas en las asociaciones palinológicas de la Formación Andapaico.

Table 1. Distribution of the palynological species identified of the Andapaico Formation.

\begin{tabular}{|c|c|c|c|c|c|c|c|}
\hline Taxones identificados/PBSJ & 374 & 361 & 362 & Taxones identificados/PBSJ & 374 & 361 & 362 \\
\hline Alisporites spp. & $\mathrm{x}$ & $\mathrm{x}$ & $\mathrm{x}$ & Cyclogranisporites mirogranus & $\mathrm{x}$ & & \\
\hline Barakarites $\mathrm{cf}$. rotatus & $\mathrm{x}$ & $\mathrm{x}$ & $\mathrm{x}$ & Hamiapollenites bullaeformis & $\mathrm{x}$ & & \\
\hline Caheniasaccites spp. & $\mathrm{x}$ & $\mathrm{x}$ & $\mathrm{x}$ & Hamiapollenites erebi & $\mathrm{x}$ & & \\
\hline Calamospora breviradiata & $\mathrm{x}$ & $\mathrm{x}$ & $\mathrm{x}$ & Hamiapollenites fusiformis & $\mathrm{x}$ & & \\
\hline Cyclogranisporites spp. & $\mathrm{x}$ & $\mathrm{x}$ & $\mathrm{x}$ & Horriditriletes $\mathrm{sp}$ & $\mathrm{x}$ & & \\
\hline Hamiapollenites ruditaeniatus & $\mathrm{x}$ & $\mathrm{x}$ & $\mathrm{x}$ & Illinites unicus & $\mathrm{x}$ & & \\
\hline Hamiapollenites spp. & $\mathrm{x}$ & $\mathrm{x}$ & $\mathrm{x}$ & Limitisporites amazoniensis & $\mathrm{x}$ & & \\
\hline Latusipollenites quadrisaccatus & $\mathrm{x}$ & $\mathrm{x}$ & $\mathrm{x}$ & Limitisporites cf. delasausei & $\mathrm{x}$ & & \\
\hline Leiotriletes directus & $\mathrm{x}$ & & $\mathrm{x}$ & Lueckisporites cf. angoulaensis & $\mathrm{x}$ & & \\
\hline Limitisporites spp. & $\mathrm{x}$ & $\mathrm{x}$ & $\mathrm{x}$ & Lueckisporites singhii & $\mathrm{x}$ & & \\
\hline Lueckisporites spp. & $\mathrm{x}$ & $\mathrm{x}$ & $\mathrm{x}$ & Lueckisporites singraulensis & $\mathrm{x}$ & & \\
\hline Lunatisporites spp. & $\mathrm{x}$ & $\mathrm{x}$ & $\mathrm{x}$ & Luckisporites stenotaeniatus & $\mathrm{x}$ & & \\
\hline Mabuitasacites crucistriatus & $\mathrm{x}$ & $\mathrm{x}$ & $\mathrm{x}$ & Lunatisporites ovatus & $\mathrm{x}$ & & \\
\hline Polarisaccites bilateralis & $\mathrm{x}$ & $\mathrm{x}$ & $\mathrm{x}$ & Lunatisporites cf. pellucidus & $\mathrm{x}$ & & \\
\hline Protohaploxypinus goraiensis & $\mathrm{x}$ & $\mathrm{x}$ & $\mathrm{x}$ & Lunatisporites cf. variesectus & $\mathrm{x}$ & & \\
\hline Protohaploxypinus spp. & $\mathrm{x}$ & $\mathrm{x}$ & $\mathrm{x}$ & Lundbladispora cf. riobonitensis & $\mathrm{x}$ & & \\
\hline Pteruchipollenites spp. & $\mathrm{x}$ & & $\mathrm{x}$ & Mabuitasaccites sp. A & $\mathrm{x}$ & & \\
\hline Punctatisporites sp. & $\mathrm{x}$ & $\mathrm{x}$ & $\mathrm{x}$ & Marsupipollenites striatus & $\mathrm{x}$ & & \\
\hline Scheuringipollenites medius & $\mathrm{x}$ & $\mathrm{x}$ & $\mathrm{x}$ & Platysaccus cf. olivae & $\mathrm{x}$ & & \\
\hline Striatopodocarpites cancellatus & $\mathrm{x}$ & $\mathrm{x}$ & $\mathrm{x}$ & Platysaccus cf. queenslandii & $\mathrm{x}$ & & \\
\hline Vittatina spp. & $\mathrm{x}$ & $\mathrm{x}$ & $\mathrm{x}$ & Potonieisporites lelei & $\mathrm{x}$ & & \\
\hline Converrucosisporites confluens & $\mathrm{x}$ & $\mathrm{x}$ & & Protohaploxypinus amplus & $\mathrm{x}$ & & \\
\hline Pakhapites fusus & $\mathrm{x}$ & $\mathrm{x}$ & & Protohaploxypinus microcorpus & $\mathrm{x}$ & & \\
\hline Pakhapites ovatus & $\mathrm{x}$ & $\mathrm{x}$ & & Retusotriletes $\mathrm{cf}$. diversiformis & $\mathrm{x}$ & & \\
\hline Pteruchipollenites gracilis & $\mathrm{x}$ & $\mathrm{x}$ & & Scheuringipollenites circularis & $\mathrm{x}$ & & \\
\hline Tuberisaccites varius & $\mathrm{x}$ & $\mathrm{x}$ & & Scheuringipollenites ovatus & $\mathrm{x}$ & & \\
\hline Accinctisporites ligatus & $\mathrm{x}$ & & & Scheuringipollenites cf. maximus & $\mathrm{x}$ & & \\
\hline Alisporites australis & $\mathrm{x}$ & & & Stellapollenites sp. & $\mathrm{x}$ & & \\
\hline Alisporites opii & $\mathrm{x}$ & & & Striapollenites sp. A & $\mathrm{x}$ & & \\
\hline Alisporites parvus & $\mathrm{x}$ & & & Striatopodocarpites gondwanensis & $\mathrm{x}$ & & \\
\hline Alisporites rioclarensis & $\mathrm{x}$ & & & Striomonosaccites cicatricosus & $\mathrm{x}$ & & \\
\hline Alisporites similis & $\mathrm{x}$ & & & Striomonosaccites ovatus & $\mathrm{x}$ & & \\
\hline Apiculatasporites sp. & $\mathrm{x}$ & & & Tornopollenites sp. & $\mathrm{x}$ & & \\
\hline Brazilea sp. A & $\mathrm{x}$ & & & Vestigisporites sp. A & $\mathrm{x}$ & & \\
\hline Brevitriletes levis & $\mathrm{x}$ & & & Vestigisporites $\mathrm{sp} . \mathrm{B}$ & $\mathrm{x}$ & & \\
\hline Caheniasaccites elongatus & $\mathrm{x}$ & & & Vitreisporites cf. signatus & $\mathrm{x}$ & & \\
\hline Cannanoropollis densus & $\mathrm{x}$ & & & Vittatina corrugata & $\mathrm{x}$ & & \\
\hline Cannanoropollis janakii & $\mathrm{x}$ & & & Vittatina costabilis & $\mathrm{x}$ & & \\
\hline Cannanoropollis mehtae & $\mathrm{x}$ & & & Vittatina fasciolata & $\mathrm{x}$ & & \\
\hline Chordasporites cf. australiensis & $\mathrm{x}$ & & & Vittatina subsaccata & $\mathrm{x}$ & & \\
\hline Colpisaccites cf. granulosus & $\mathrm{x}$ & & & Vittatina vittifera & $\mathrm{x}$ & & \\
\hline Corisaccites alutas & $\mathrm{x}$ & & & Weylandites lucifer & $\mathrm{x}$ & & \\
\hline Corisaccites vanus & $\mathrm{x}$ & & & Weylandites magmus & $\mathrm{x}$ & & \\
\hline Crucisaccites latisulcatus & $\mathrm{x}$ & & & Caheniasaccites flavatus & & $\mathrm{x}$ & \\
\hline Crutaesporites globosus & $\mathrm{x}$ & & & Caheniasaccites ovatus & & $\mathrm{x}$ & \\
\hline Crutaesporites sp. A & $\mathrm{x}$ & & & Vallatisporites arcuatus & & $\mathrm{x}$ & \\
\hline Cycadopites crassimarginis & $\mathrm{x}$ & & & Lundbladispora cf. brasiliensis & & & $\mathrm{x}$ \\
\hline Cycadopites follicularis & $\mathrm{x}$ & & & & & & \\
\hline
\end{tabular}


Descripción. Grano de polen monosacado, de simetría bilateral, contorno general circular a oval, elongado en sentido transversal. Cuerpo central subcircular a oval-transverso. Adherencia del saco proximal-ecuatorial y distal-bilateral paralela a cóncava, que se continúan hasta el borde del saco. Dimensiones. (10 ejs.). Longitud total 36(40,6)52 $\mu \mathrm{m}$; cuerpo $24(30,3) 40 \mu \mathrm{m}$. Ancho total $38(45,2) 58$ $\mu \mathrm{m}$; cuerpo $22(27,6) 35 \mu \mathrm{m}$. Adherencia distal del saco $12(17,3) 21 \mu \mathrm{m}$.

Infraturma DIPOLSACCITI Hart emend. Dibner, 1971

Género Tuberisaccites Lele \& Makada, 1972

Especie tipo. Tuberisaccites varius Lele \& Makada, 1972.

Tuberisaccites varius Lele \& Makada, 1972

(Figura 4G)

Descripción. Grano de polen monosacado de contorno subcircular. Cuerpo central de contorno subcircular con 8 protuberancias (uno central y siete periféricas). Saco angosto con pliegues radiales y márgenes ondulados.

Dimensiones. ( 1 ej.). Diámetro total $132 \mu \mathrm{m}$; cuerpo $90 \mu \mathrm{m}$; protuberancias 15-22 $\mu \mathrm{m}$.

Infraturma STRIATORNATI Jansonius, 1962

Crustaesporites Leschik emend. Jansonius, 1962

Especie tipo. Crustaesporites globosus Leschik, 1956.

Crutaesporites sp. A

(Figura 3O)

Descripción. Grano de polen monosacado de contorno subtriangular trilobado. Cuerpo central subcircular. Cara proximal con 2-4 tenias irregulares, a veces pobremente definidas. Saco irregular adherido subecuatorialmente en ambas caras, con 1 a 3 constricciones. Pueden observarse pliegues radiales.

Dimensiones. (22 ejs.). Diámetro total: mayor 75(102,2)125 $\mu \mathrm{m}$; menor 65(81,2)101 $\mu \mathrm{m}$; cuerpo 45(76,2)90 $\mu \mathrm{m}$. Tenias 4-8 $\mu \mathrm{m}$.

Comentarios. Crutaesporites sp. A se diferencia de $C$. globosus Leschik (1956) por sus dimensiones y número de tenias. Los ejemplares identificados como C. globosus presentan las siguientes dimensiones: diámetro total: 66-76 $\mu \mathrm{m}$; diámetro del cuerpo central: 48-64 $\mu \mathrm{m}$; además el número de tenias varía de 8 a 11 .

Infraturma STRIASACCITES Bharadwaj, 1962

Mabuitasaccites Bose \& Kar, 1966

Especie tipo. Mabuitasaccites ovatus Bose \& Kar, 1966.

Mabuitasaccites crucistriatus (Ybert)

Playford \& Dino, 2000b

(Figura $3 \mathrm{~W}$ )
Tabla 2. Composición genérica de las asociaciones palinológicas de la Formación Andapaico, agrupadas según la afinidad botánica (AB) de sus integrantes (cf. Balme, 1995): Sp, Sphenophyta; Pt, Pteridophyta; Ly, Lycophyta; Gy, Gymnospermae; Co, Coniferophyta; GI, Glossopteridales; Cay, Caytoniales; Pel, Peltaspermales; F, Fungii; ETL, esporas trilete lisas; ETA, esporas triletes apiculadas; ETCgz, esporas trilete cingulizonadas; GPM, granos de polen monosacados; GPB, granos de polen bisacados lisos; GPE, granos de polen Estriados; GPT, granos de polen teniados; $\mathbf{n}$, cantidad de ejemplares.

Table 2. Generic composition of the palynological assemblages of the Andapaico Formation, by botanical affinity (AB) of this members (cf. Balme, 1995): Sp, Sphenophyta; Pt, Pteridophyta; Ly, Lycophyta; Gy, Gymnosperms; Co, Coniferophyta; GI, Glossopteridales; Cay, Caytoniales; Pel, Peltaspermales; F, Fungii; ETL, laevigate trilete spores; ETA, apiculate trilete spores; ETCgz, cingulizonate trilete spores; GPM, monosaccate pollen grains; GPB, bisaccate pollen grains; GPE, striate pollen grains; GPT, taeniate pollen grains; $\mathbf{n}$, number of specimens.

\begin{tabular}{|c|c|c|c|c|c|c|c|}
\hline \multirow{2}{*}{$\begin{array}{l}\text { PBSJ } \\
\text { Taxones }\end{array}$} & \multicolumn{2}{|c|}{374} & \multicolumn{2}{|c|}{361} & \multicolumn{2}{|c|}{362} & \multirow{2}{*}{$\mathrm{AB}$} \\
\hline & $\mathrm{n}$ & $\%$ & $\mathrm{n}$ & $\%$ & $\mathrm{n}$ & $\%$ & \\
\hline Calamospora & 3 & 0,4 & 4 & 2 & - & - & $\mathrm{Sp}$ \\
\hline Leiotriletes & 2 & 0,3 & 4 & 2 & 4 & 1,2 & $\mathrm{Pt}$ \\
\hline Punctatisporites & 21 & 2,7 & 31 & 15,5 & 6 & 1,8 & $\mathrm{Pt}$ \\
\hline ETL indet. & 32 & 4,2 & 25 & 12,5 & 15 & 4,5 & $\mathrm{Pt}$ \\
\hline Converrucosisporites & 2 & 0,3 & 1 & 0,5 & 1 & 0,3 & $\mathrm{Pt}$ \\
\hline Cyclogranisporites & 7 & 0,9 & 1 & 0,5 & 4 & 1,2 & $\mathrm{Pt}$ \\
\hline ETA indeterminados & 9 & 1,2 & 2 & 1 & 3 & 0,9 & $\mathrm{Pt}$ \\
\hline Vallatisporites & 3 & 0,4 & - & - & - & - & Ly \\
\hline Lundbladispora & - & - & 3 & 1,5 & 1 & 0,3 & Ly \\
\hline Vallatisporites & 43 & 5,6 & 1 & 0,5 & 1 & 0,3 & Ly \\
\hline ETCgz indeterminados & 1 & 0,1 & - & - & - & - & Ly \\
\hline Barakarites & 6 & 0,8 & 1 & 0,5 & 3 & 0,9 & $\mathrm{Co} ?$ \\
\hline Caheniasaccites & 3 & 0,4 & 1 & 0,5 & 9 & 2,7 & $\mathrm{Co}$ \\
\hline Cannanoropollis & 6 & 0,8 & 6 & 3 & 14 & 4,2 & Co \\
\hline Crucisaccites & 3 & 0,4 & - & - & - & - & Gy \\
\hline Latusipollenites & 9 & 1,2 & 1 & 0,5 & 10 & 3 & Gy \\
\hline Mabuitasaccites & 35 & 4,5 & 5 & 2,5 & 16 & 4,8 & Gy \\
\hline Striomonosaccites & 1 & 0,1 & - & - & - & - & Gy \\
\hline Potonieisporites & - & - & 1 & 0,5 & 2 & 0,6 & Gy \\
\hline Tubersicacites & 1 & 0,1 & - & - & - & - & Gy \\
\hline GPM indeterminados & 23 & 3 & 19 & 9,5 & 68 & 20,3 & \\
\hline Colpisaccites & 3 & 0,4 & - & - & - & - & Co \\
\hline Limitisporites & 2 & 0,3 & 2 & 1 & 2 & 0,6 & Co \\
\hline Platysaccus & 3 & 0,4 & 1 & 0,5 & 1 & 0,3 & Co \\
\hline Scheuringipollenites & 34 & 4,4 & 5 & 2,5 & 2 & 0,6 & $\mathrm{Co}$ \\
\hline Vestigisporites & 3 & 0,4 & - & - & 1 & 0,3 & $\mathrm{Co} ?$ \\
\hline Alisporites & 50 & 6,5 & 8 & 4 & 24 & 7,2 & Cay \\
\hline Pteruchipollenites & 10 & 1,3 & 3 & 1,5 & 6 & 1,8 & Cay \\
\hline Vietrisporites & 1 & 0,1 & - & - & - & - & Cay \\
\hline GPB indeterminados & 64 & 8,3 & 15 & 7,5 & 36 & 10,7 & $\mathrm{Co}$ \\
\hline Corisaccites & 8 & 1 & - & - & - & - & Co \\
\hline Lueckisporites & 10 & 1,3 & - & - & - & - & Co \\
\hline Lunatisporites & 1 & 0,1 & - & - & - & - & Co \\
\hline Pakhapites & 9 & 1,2 & 1 & 0,5 & - & - & Co \\
\hline Hamiapollenites & 48 & 6,2 & 4 & 2 & 7 & 2,1 & Gy \\
\hline Illinites & 14 & 1,8 & - & - & 3 & 0,9 & Gy \\
\hline Marsupipollenites & 4 & 0,5 & 1 & 0,5 & 3 & 0,9 & Gl \\
\hline Protohaploxypinus & 61 & 7,9 & 9 & 4,5 & 14 & 4,2 & Gl \\
\hline Striatopodocarpites & 13 & 1,6 & 5 & 2,5 & 4 & 1,2 & Gl \\
\hline Weylandites & 7 & 0,9 & - & - & - & - & Gl \\
\hline Vittatina & 101 & 13,1 & 9 & 4,5 & 17 & 5,1 & Pel \\
\hline GPE indeterminados & 104 & 13,5 & 28 & 14 & 51 & 15,2 & \\
\hline Cycadopites & 4 & 0,5 & - & - & - & - & Pel \\
\hline Polarisaccites & 2 & 0,3 & - & - & 4 & 1,2 & Gy \\
\hline Fungii indeterminados & 4 & 0,5 & 2 & 1 & 3 & 0,9 & $\mathrm{~F}$ \\
\hline Total & 770 & 100 & 200 & 100 & 335 & 100 & \\
\hline
\end{tabular}


Tabla 3. Composición de las microfloras identificadas en la Formación Andapaico, según sus posibles afinidades botánicas y agrupadas en categorías supragenéricas (CS): EM, esporas monoletes; ET, esporas triletes; GPM, granos de polen monosacados; GPB, granos de polen bisacados lisos; GPT, granos de polen teniados; GPS, granos de polen sulcados; GPP, granos de polen polisacados.

Table 3. Microflora composition in Andapaico Formation identified as potential botanical affinities, and grouped into suprageneric categories (CS): EM, monolete spores; ET, trilete spores; GPM, monosaccates pollen grains; GPB, bisaccate pollen grains; GPT, taeniate pollen grains; GPS, sulcate pollen grains; GPP, polysacate pollen grains.

\begin{tabular}{llccc}
\hline CS & Grupo parental / PBSJ & 374 & 361 & 362 \\
\hline EM & Sphenophyta & 0,4 & 2 & - \\
\hline \multirow{2}{*}{ ET } & Pteridophyta & 9,5 & 32,5 & 9,8 \\
& Lycophyta & 6,1 & 2 & 0,6 \\
& & 16 & 36,5 & 10,4 \\
\hline \multirow{4}{*}{ GPM } & Gymnospermas & 6,4 & 3,5 & 8,4 \\
& Coniferopsida & 1,9 & 4 & 7,8 \\
& Indeterminados & 3 & 9,5 & 20,3 \\
& & 11,3 & 17 & 36,4 \\
\hline \multirow{4}{*}{ GPB } & Coniferopsidal & 5,8 & 4 & 1,8 \\
& Caytoniales & 7,9 & 5,5 & 9 \\
& Indeterminados & 8,3 & 7,5 & 10,7 \\
& & 22 & 17 & 21,5 \\
\hline \multirow{4}{*}{ Total } & Gymnospermas & 8,1 & 2 & 3 \\
& Coniferopsida & 3,6 & 0,5 & - \\
GPT & Peltaspermales & 13,2 & 4,5 & 5,1 \\
& Glossopteridales & 11 & 7,5 & 6,3 \\
& Indeterminados & 13,5 & 14 & 15,2 \\
& & 49,4 & 28,5 & 29,6 \\
\hline GPS & Peltaspemales & 0,5 & - & - \\
\hline GPP & Gymnospermas & 0,3 & - & 1,2 \\
\hline Fungii & & 0,5 & 1 & 0,9 \\
\hline
\end{tabular}

1997 Mabuitasaccites ovatus Bose \& Kar; Ghavidel-Syooki, pl. IV, figs. 5, 11.

Descripción. Grano de polen monosacado de contorno subcircular a ovalado por deformación. Cuerpo central subcircular, con tenias transversales ahusadas, algunas incompletas acuñadas entre las mayores. Una de las caras

del cuerpo porta 8-16 tenias y la opuesta, 6-10, dispuestas en sentido perpendicular a oblicuo respecto a las primeras. Saco angosto.

Dimensiones. (42 ejs.). Diámetro total, 60(75,9)100 $\mu \mathrm{m}$; cuerpo 45(63)80 $\mu \mathrm{m}$. Ancho de las tenias 2-6 $\mu \mathrm{m}$.

\section{Mabuitasaccites sp. A}

(Figura 4A)

Descripción. Grano de polen monosacado de contorno subcircular. Cuerpo central teniado, de contorno subcircular a ovalado. Una de las caras del cuerpo porta de 7 a 9 tenias y la opuesta de 4 a 6 , éstas se disponen perpendicular a oblicuamente a las primeras y paralelo al mayor desarrollo del grano. Las tenias son completas, de ancho constante (varían entre 7-12 $\mu \mathrm{m})$, separadas entre sí por angostas calles. Saco angosto.

Dimensiones. ( 1 ej.). Diámetro total $80 \times 101 \mu \mathrm{m}$; cuerpo central $50 \times 75 \mu \mathrm{m}$.
Comentarios. La cantidad de tenias, sus dimensiones y disposiciones, permiten diferenciar a Mabuitasaccites sp. A del resto de las especies del género.

\section{Subturma DISACCITES Cookson, 1947 Infraturma STRIATITI Pant, 1954}

Hamiapollenites Wilson emend. Tschudy \& Kosanke, 1966

Especie tipo. Hamiapollenites saccatus Wilson, 1962.

Hamiapollenites bullaeformis (Samoilovich) Jansonius, 1962 (Figura 4Y)

Descripción. Grano de polen bisacado, teniado, de contorno oval longitudinal. Cuerpo central de exina gruesa, oscuro. Capa con 9-12 tenias trasversales, mayormente completas, aunque algunas acuñadas entre las mayores. Ancho de las tenias, 4-5 $\mu \mathrm{m}$. Cápula lisa con una franja engrosada de hasta $1 / 3$ de ancho respecto del cuerpo, de forma subrectangular a ahusada, que bisecta longitudinalmente al cuerpo. Sacos iguales a mayores a $1 / 2$ círculo, de inserción proximal ecuatorial y distal subecuatorial, levemente inclinados.

Dimensiones. (5 ejs.). Ancho total 61(67,6)90 $\mu \mathrm{m}$; cuerpo $35(43) 49 \mu \mathrm{m}$; saco 10(13,8)22 $\mu \mathrm{m}$. Largo: cuerpo 51(57,6)62 $\mu \mathrm{m}$; saco $32(41,6) 51 \mu \mathrm{m}$.

\section{Hamiapollenites erebi Utting, 1994} (Figura 4U)

Descripción. Grano de polen bisacado, teniado, de contorno oval longitudinal, haploxilonoide a levemente diploxilonoide. Cuerpo central subrectangular a oval transversal. Capa con 2 a 3 tenias trasversales oscuras, completas, de ancho constante (7-10 $\mu \mathrm{m})$. Cápula lisa, de márgenes paralelos, con una franja engrosada de hasta $1 / 3$ de ancho respecto del cuerpo, de forma subrectangular, que bisecta longitudinalmente al cuerpo. Sacos mayores a $1 / 2$ círculo, de inserción proximal ecuatorial y distal subecuatorial con pliegues asociados.

Dimensiones. ( 3 ejs.). Ancho total 70(72)75 $\mu \mathrm{m}$; cuerpo 20(32)38 $\mu \mathrm{m}$; engrosamiento distal 12-16 $\mu \mathrm{m}$; saco 19(26,6)35 $\mu \mathrm{m}$. Longitud: cuerpo $30(35,6) 40 \mu \mathrm{m}$; saco $28(33,6) 42 \mu \mathrm{m}$.

\section{Hamiapollenites ruditaeniatus Qu \& Wang, 1986} (Figuras 4V-X)

Comentarios. Hamiapollenites ruditaeniatus incluye a formas haploxilonoides, ovaladas elongadas en sentido transversal. En los ejemplares aquí estudiados se observa una variación morfológica gradual respecto del contorno subrectangularoval trasversal a formas de contorno general subcircular, con una relación ancho/largo del grano de 1,0 a 1,8. Como la gradación entre la forma del cuerpo y la general es continua en la población aquí estudiada, entendemos que todos los ejemplares deben ser incluidos en la misma especie.

Comparaciones. Hamiapollenites ruditaeniatus y H. erebi Utting se caracterizan por ser las especies del género con el menor número de tenias proximales. Sin embargo, H. ruditaeniatus 

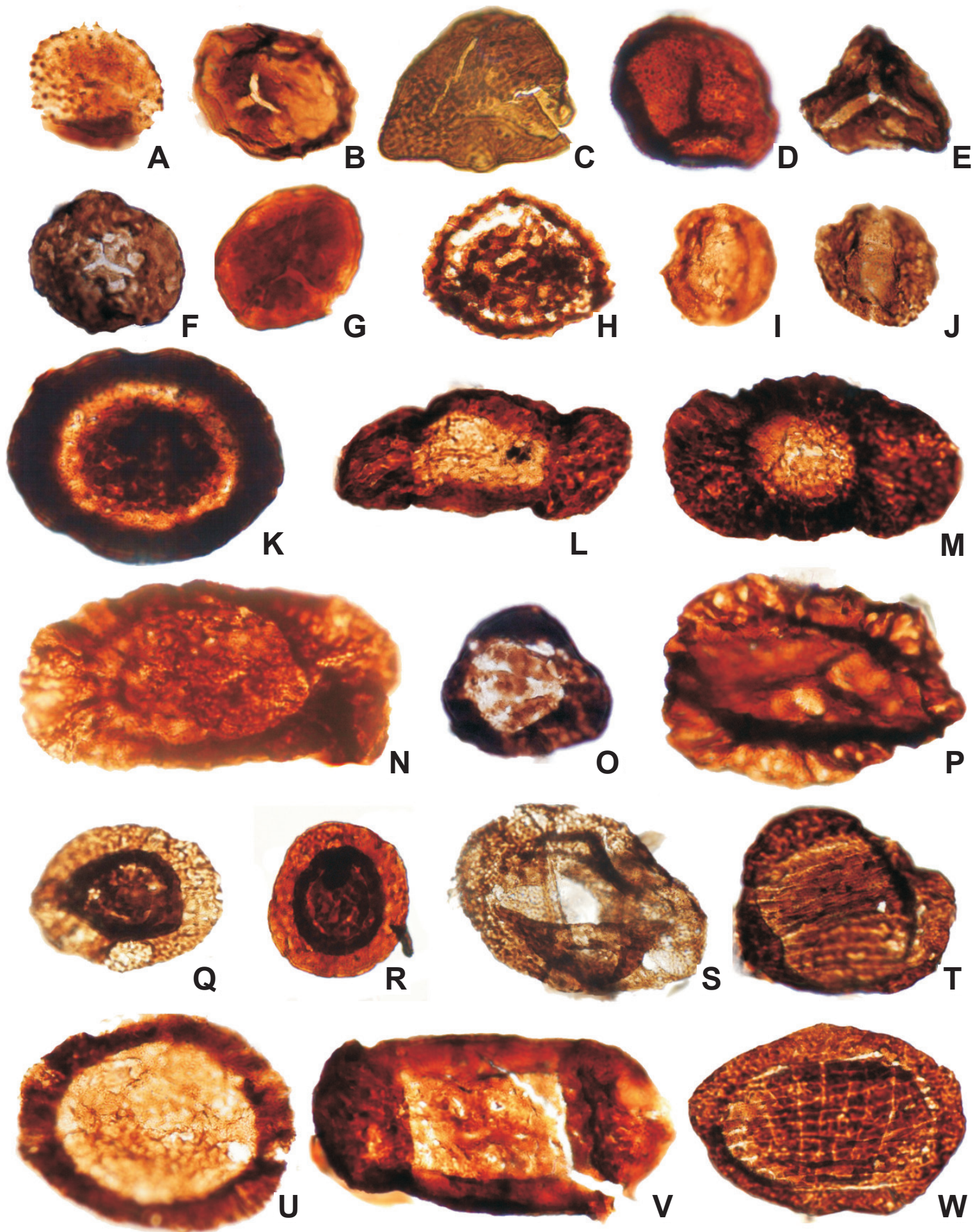

Figura/Figure 3. A, Brevitriletes levis (Balme \& Hennelly) Bharadwaj \& Srivastava, 1969. PBSJ 374(20) M49/1; B, Calamospora breviradiata Kosanke, 1950. PBSJ 374(8) D41/0; C, Converrucosisporites confluens (Archangelsky \& Gamerro) Playford \& Dino, 2002. PBSJ 361/483(3) U50/4; D. Cyclogranisporites microgranus (Balme \& Hennelly) Playford \& Dino, 2002. PBSJ 374(12) K60/0; E, Leiotriletes directus Balme \& Hennelly, 1956. PBSJ 374(19) U56/0; F, Punctatisporites sp. PBSJ 374(4) N53/3; G, Retusotriletes sp. cf. R. diversiformis (Balme \& Hennelly) Balme \& Playford, 1967 PBSJ 374(20) N53/3; H, Vallatisporites arcuatus (Marques-Toigo) Archangelsky \& Gamerro, 1979. PBSJ 374(15) Z53/0; I-J, Accinctisporites ligatus Leschik, 1955, en Kräusel \& Leschik, 1955; I, PBSJ 374(6) F52/2; J, PBSJ 374(6) V35/1; K, Barakarites sp. cf. B. rotatus (Balme \& Hennelly) Bharadwaj \& Tiwari, 1964. PBSJ 374 (13) K48/2; L, Caheniasaccites elongatus Bose \& Kar, 1966. PBSJ 374(15) X48/2; M, Caheniasaccites flavatus Bose \& Kar, 1966. PBSJ 374(16) Y58/3; N, Caheniasaccites ovatus Bosé \& Kar emend. Gutiérrez, 1993. PBSJ 374(15) Q61/4; O, Crutaesporites sp. A. PBSJ 374(4) F42/0; P, Cannanoropollis mehtae (Lele) Bose \& Maheshwari, 1968. PBSJ 374(10) M60/3; Q-R, Circumplicatipollis plicatus Ottone \& Azcuy, 1988; Q, PBSJ 374(4) C50/0; R, PBSJ 374(12) L47/4; S, Crucisaccites latisulcatus Lele \& Maithy, 1964. PBSJ 374 (2) P42/1; T, Crutaesporites globosus Leschik, 1956. PBSJ 374(18) F68/1; U, Cannanoropollis janakii Potonié \& Sah, 1960. PBSJ 374(10) P46/0; V, Latusipollenites quadrisaccatus Marques-Toigo, 1974. PBSJ 374(9) C47/4; W, Mabuitasaccites crucistriatus (Ybert) Playford \& Dino, 2000b. PBSJ 374(19) Z54/3. Esporas: A-H, X750; granos de polen: I-W X500. / Spores: A-H, X750; pollen grains: I-W, X500. 

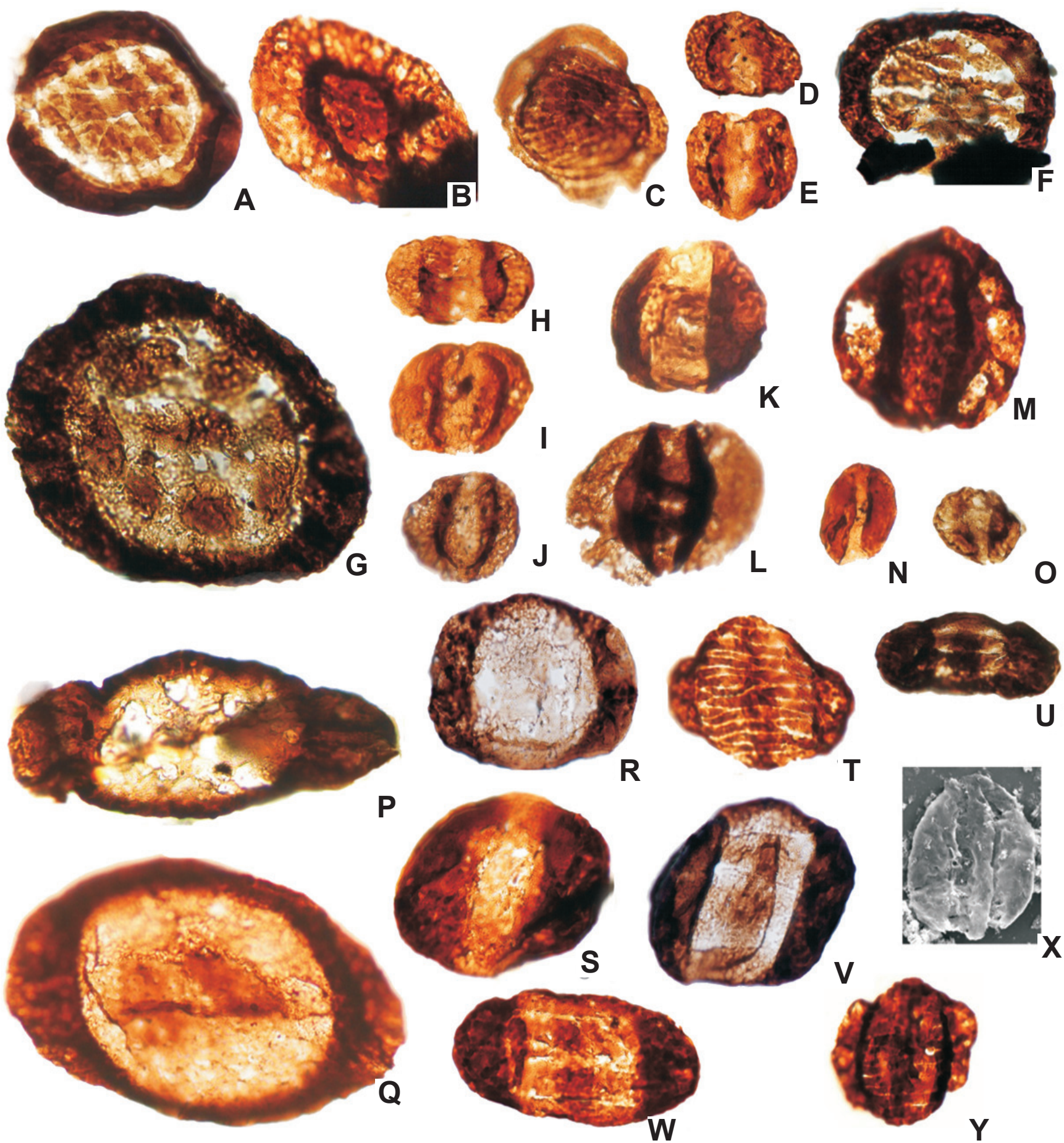

Figura/Figure 4. A, Mabuitasaccites sp. A. PBSJ 374(4) T24/0; B, Potonieisporites lelei Maheshwari, 1967. PBSJ 374(15) G38/2; C, Striomonosaccites cicatricosus Archangelsky \& Gamerro, 1979. PBSJ 374(21) P63/3; D, Alisporites australis de Jersey, 1962. PBSJ 374(19) R54/3; E, Alisporites opii Daugherty, 1941. PBSJ 374(20) S65/4; F, Striomonosaccites ovatus Bharadwaj, 1962. PBSJ 374(3) R34/1; G, Tuberisaccites varius Lele \& Makada, 1972. PBSJ 374(4) P44/0; H, Alisporites rioclarensis Menéndez, 1976 PBSJ 374(10) L42/3; I, Alisporites parvus de Jersey, 1962. PBSJ 374(13) Z57/0; J, Alisporites similis Balme 1957. PBSJ 374(13) N60/4; K, Colpisaccites sp. cf. C. granulosus Archangelsky \& Gamerro, 1979. PBSJ 374(4) C53/0; L, Limitisporites amazoniensis Playford \& Dino, 2000b PBSJ 374(16) E37/4; M, Limitisporites sp. cf. L. delasausei Schaarschmidt, 1963. PBSJ 374(15) E64/3; N, Scheuringipollenites medius (Burjack) Dias-Fabrício, 1981. PBSJ 374(10) L34/4; O, Vitreisporites sp. cf. V. signatus Leschik, 1955. PBSJ 374(2) E37/4; P, Vestigisporites sp. A. PBSJ 374(11) N42/3; Q, Vestigisporites sp. B. PBSJ 374(12) Z47/3; R, Pteruchipollenites gracilis (Segroves) Foster, 1979. PBSJ 374(7) O53/0; S, Scheuringipollenites sp. cf. S. maximus (Hart) Tiwari, 1973. PBSJ 374(10) B45/0; T, Hamiapollenites fusiformis Marques-Toigo emend. Archangelsky \& Gamerro, 1979. PBSJ 374(19) O42/0; U, Hamiapollenites erebi Utting, 1994. PBSJ 374(7) O53/3; V-X, Hamiapollenites ruditaeniatus Qu \& Wang, 1986; V, PBSJ 374(4) P35/0; W, PBSJ 374(18) E50/4; X, PBSJ 374 MEB; Y, Hamiapollenites bullaeformis (Samoilovich) Jansonius, 1962. PBSJ 374(9) P30/0. Todos X500 excepto U X750. / All X500 except U X750. 
se diferencia por su tamaño mayor, contorno general oval transversal haploxilonoide, engrosamiento distal menor (hasta $1 / 4$ del ancho respecto del cuerpo), cápula de lados rectos a cóncavos y sacos iguales a menores a $1 / 2$ círculo.

Dimensiones. (15 ejemplares). Ancho: total 71(91,5)123 $\mu \mathrm{m}$; cuerpo 50(65,5)89 $\mu \mathrm{m}$; ancho de las tenias proximales 6-20 $\mu \mathrm{m}$; engrosamiento distal 5-18 $\mu \mathrm{m}$. Saco: inserción proximal $7(13,2) 21 \mu \mathrm{m}$; inserción distal 15(20)38 $\mu \mathrm{m}$. Longitud: cuerpo $44(53,4) 69 \mu \mathrm{m}$; saco $42(67,4) 87 \mu \mathrm{m}$.

\section{Corisaccites Venkatachala \& Kar, 1966}

Especie tipo. Corisaccites alutas Venkatachala \& Kar, 1966.

\section{Corisaccites vanus Venkatachala \& Kar, 1966}

(Figuras 5D-E)

Descripción. Grano de polen bisacado, haploxilonoide, teniado, de contorno general oval (en sentido longitudinal o transversal) a subcircular. Cuerpo central circular, capa con una profunda estría ecuatorial transversal que divide la cara proximal en 2 anchas tenias equidimensionales, subrectangulares a reniformes, de ancho contante (10-28 $\mu \mathrm{m}$ ). Cápula de lados paralelos a cóncavos (hasta $1 / 3$ de la longitud del grano). Sacos menores o iguales a $1 / 2$ círculo contactados en ambos extremos, conformando amplios puentes sobre la cara proximal, lo que le otorga en algunos casos un aspecto monosacado. Adherencia proximal ecuatorial y distal bilateral.

Dimensiones. (46 ejs.). Longitud total 40(64,5)82 $\mu \mathrm{m}$; cuerpo 25(42,4)59 $\mu \mathrm{m}$. Ancho total 42(67,6)95 $\mu \mathrm{m}$; cuerpo $27(43,9) 60 \mu \mathrm{m}$.

Comentarios y discusión. La diagnosis de Corisaccites vanus Venkatachala \& Kar (1966) incluye granos bisacados haploxilonoides, de cuerpo oval transversal con un sulco (cápula?) simple de ancho uniforme. C. alutas Venkatachala \& Kar (1966), se diferencia por su cuerpo de contorno subcircular a oval longitudinal y no presentar un aspecto general monosacado. En los ejemplares aquí estudiados, se ha observado una variación morfológica gradual respecto del mayor desarrollo del cuerpo central, incluyendo desde formas ovales en sentido transversal (Figura 5D) hasta ovales longitudinalmente (Figura 5E). La relación entre el ancho y la longitud del grano, varía transicionalmente entre 0,9-1,4, y la relación entre el ancho del cuerpo central y el total gradúa entre $0,5-0,9$. No se observan poblaciones independientes del conjunto, lo que permite considerar a las todas formas comprendidas entre ambos tipos morfológicos (oval/longitudinal a oval/transverso) como ejemplares de una misma especie.

También fueron comparados los ejemplares con diversos morfotipos de Illinites unicus (Azcuy et al., 2002), aunque esta especie presenta una estría central subecuatorial, de la cual se ramifican estrías menores generando una división del cuerpo asimétrica. En los ejemplares aquí estudiados se observa una calle central horizontal ecuatorial, que bisecta al cuerpo generando 2 tenias equidimensionales, sin divisiones menores.
Lueckisporites Potonié \& Klaus emend. Klaus, 1963

Especie tipo. Lueckisporites virkkiae Potonié \& Klaus, 1954.

Lueckisporites singhii Balme, 1970

(Figuras 5F-G)

Descripción. Grano de polen bisacado, teniado, haploxilonoide, de contorno general oval transversal. Cuerpo central oval longitudinal, de contorno poco definido. Capa bisectada por una estría transversal, que la divide proximalmente en dos tenias equidimensionales (20-22 $\mu \mathrm{m}$ de ancho). Cápula ancha (hasta la mitad del ancho del cuerpo), de lados cóncavos hacia el centro. Sacos iguales a 1/2 círculo, de inserción proximal ecuatorial y distal bilateral.

Dimensiones. (2 ejs.). Ancho: total $98 \mu \mathrm{m}$; saco: $35 \mu \mathrm{m}$. Longitud de cuerpo $52 \mu \mathrm{m}$.

\section{Lueckisporites singrauliensis Sinha, 1972}

(Figura 5I)

Descripción. Grano de polen bisacado, teniado, fuertemente diploxilonoide. Cuerpo central subcircular, pequeño, de hasta 2/3 de largo respecto del largo total. Capa bisectada por una estría transversal de lados paralelos a cóncavos que delimita 2 tenias reniformes. Cápula de $1 / 3$ a 2/3 de ancho del cuerpo, de lados rectos a cóncavos. Sacos mayores a 1/2 círculo, de adherencia proximal ecuatorial y distal bilateral, con un denso infrareticulado.

Dimensiones. (5 ejs.). Ancho: total 52(66,5)97 $\mu \mathrm{m}$; cuerpo 25(33,4)49 $\mu \mathrm{m}$; tenias 10-18 $\mu \mathrm{m}$. Inserción distal del saco $21(28) 40 \mu \mathrm{m}$; longitud: cuerpo $20(30) 40 \mu \mathrm{m}$; saco $45(55,2) 82 \mu \mathrm{m}$.

Comparaciones. Lueckisporites singrauliensis Sinha incluye formas fuertemente diploxilonoides, de cuerpo central pequeño, subcircular y la cápula angosta de lados rectos. L. virkkiae (Potonié \& Klaus) Klaus si bien comprende formas que varían el grado diploxilonoide del contorno, el cuerpo central es oval trasversal y la cápula es ancha, de lados cóncavos.

Protohaploxypinus Samoilovich emend. Morbey, 1975

Especie tipo. Protohaploxypinus latissimus (Luber) Samoilovich, 1953.

Protohaploxypinus microcorpus (Schaarschmidt) Clarke, 1965 (Figura 5M)

Descripción. Grano de polen bisacado teniado haploxilonoide. Contorno general oval-transverso a ligeramente subrectangular. Cuerpo central subcircular a levemente oval en sentido longitudinal, de márgenes poco discernibles. Capa con 7 a 10 tenias trasversales pobremente definidas completas e incompletas. Cápula de $4 / 5$ del ancho del cuerpo de márgenes levemente cóncavos. Sacos iguales a menores de 1/2 círculo unidos proximalmente por delgados puentes exinales. Adherencia proximal ecuatorial y distal bilateral. 

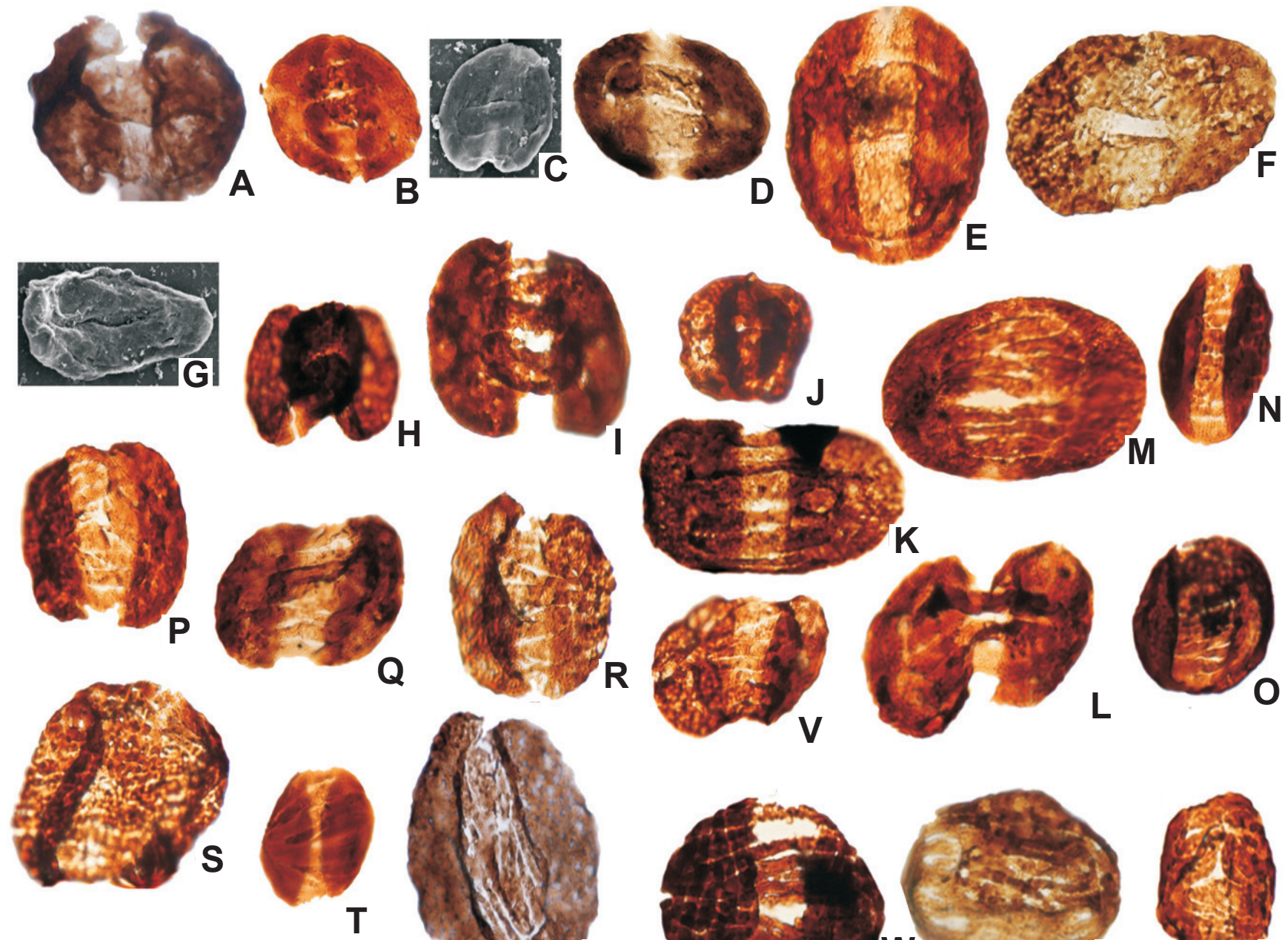

Q
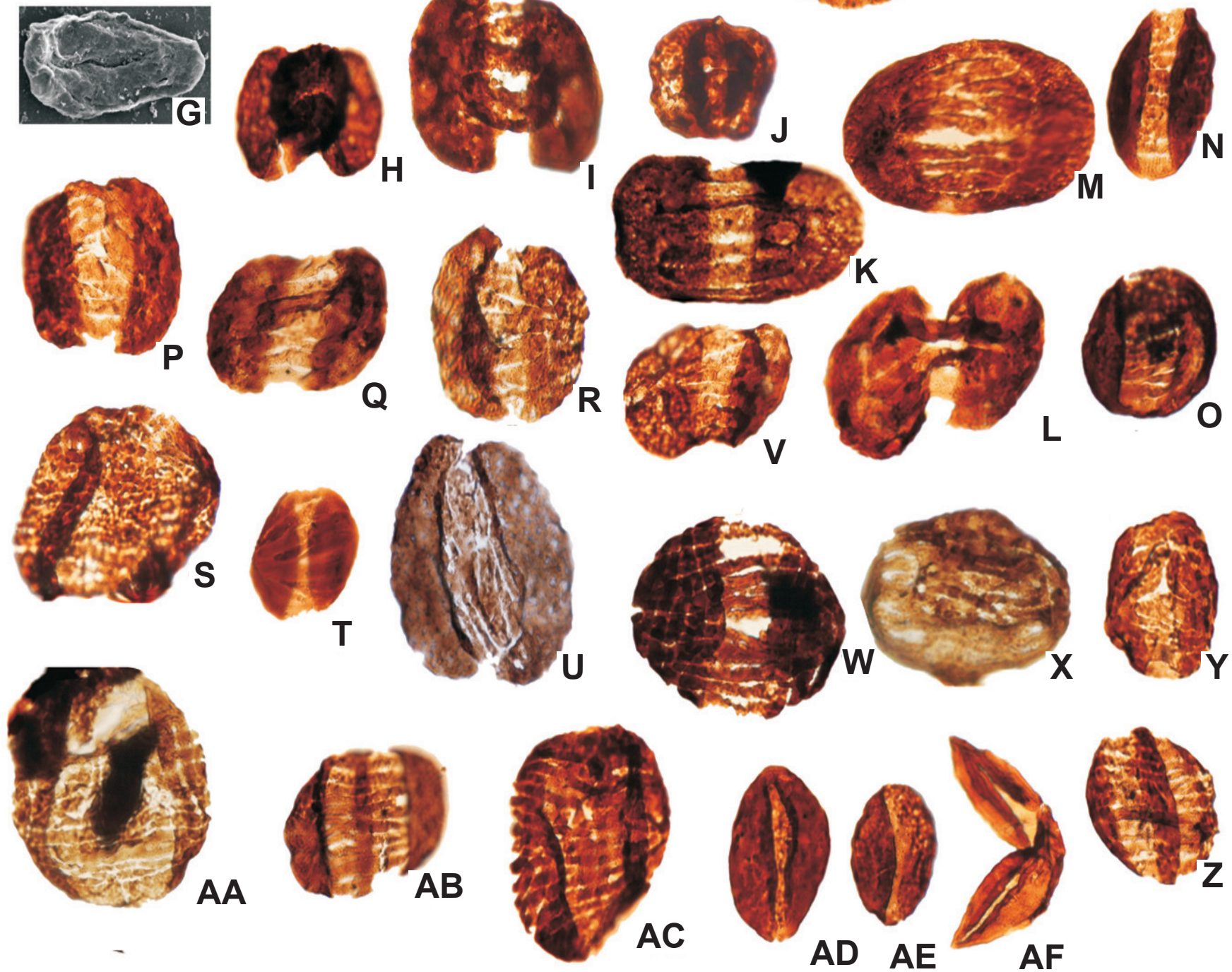

Figura/Figure 5. A, Corisaccites alutas Venkatachala \& Kar, 1966. PBSJ 374(4) E53/1; B-C, Illinites unicus Kosanke emend. Jansonius \& Hills, 1976; B, PBSJ 374(20) U65/1; C, PBSJ 374MEB; D, E, Corisaccites vanus Venkatachala \& Kar, 1966. D, PBSJ 374(5) N41/3; E, PBSJ 374(13) S35/1; F-G, Lueckisporites singhii Balme, 1970. F, PBSJ 374(2) D53/4; G, PBSJ 374MEB; H, Lueckisporites sp. cf. L. angoulaensis Jardiné 1974. PBSJ 374(9) M54/2; I, Lueckisporites singrauliensis Sinha, 1972. PBSJ 374(21) S55/1; J, Lunatisporites ovatus (Goubin) Maheshwari \& Banerjee, 1975. PBSJ 374(17) D58/2; K, Lunatisporites sp. cf. L. pellucidus (Goubin) Maheshwari \& Banerjee, 1975 PBSJ 374(21) J55/2; L, Lueckisporites stenotaeniatus Menéndez, 1976. PBSJ 374(17) J61/0; M, Protohaploxypinus microcorpus (Schaarschmidt) Clarke, 1965. PBSJ 374(13) U57/0; N, Pakhapites fusus (Bose \& Kar) Menéndez, 1971. PBSJ 374(16) R44/0; O, Protohaploxypinus goraiensis (Potonié \& Lele) Hart, 1964. PBSJ 374(13) F35/0; P, Lunatisporites sp. cf. L. variesectus Archangelsky \& Gamerro, 1979. PBSJ 374(15) E39/2; Q, Protohaploxypinus amplus (Balme \& Hennelly) Hart, 1964. PBSJ 374(12) E60/0; R, Striatopodocarpites gondwanensis Lakhanpal, Sah \& Dube, 1960. PBSJ 374(18) B36/2; S, Vittatina corrugata Marques-Toigo, 1974. PBSJ 374(19) X36/0; T, Pakhapites ovatus (Bose \& Kar) García, 1996. PBSJ 374(5) S51/1; U, Striapollenites sp. A. PBSJ 374(4) J26/0; V, Striatopodocarpites cancellatus (Balme \& Hennelly) Hart, 1965. PBSJ 374(20) X41/2; W, Weylandites magmus (Bose \& Kar) Backhouse, 1991. PBSJ 374(14) X65/0; X, Vittatina vittifera (Luber \& Waltz) Samoilovich, 1953. PBSJ 374(11) C38/1; Y, Marsupipollenites striatus (Balme \& Hennelly) Hart, 1965. PBSJ 374(13) F42/1; Z, Vittatina costabilis Wilson, 1962. PBSJ 374(14) D34/2; AA, Vittatina fasciolata (Balme \& Hennelly) Bharadwaj, 1962. PBSJ 374(11) P55/4; AB, Vittatina subsaccata Samoilovich, 1953. PBSJ 374(1) W39/1; AC, Weylandites lucifer (Bharadwaj \& Salujha) Foster, 1975. PBSJ 374(18) V51/1; AD, Cycadopites crassimarginis (de Jersey) de Jersey, 1964. PBSJ 374(20) N53/4; AE, Cycadopites follicularis Wilson \& Webster, 1946. PBSJ 374(17) C48/2; AF, Brazilea sp. A Playford \& Dino, 2000b. PBSJ 374(18) M53/3. Todos X 500 excepto C X750. / All X500 except C X750. 
Dimensiones. ( 6 ejs.). Ancho: total 52(66,5)97 $\mu \mathrm{m}$; cuerpo $25(33,4) 49 \mu \mathrm{m}$; tenias $2-4 \mu \mathrm{m}$. Inserción distal del saco $21(28) 40$ $\mu \mathrm{m}$; longitud: cuerpo 20(30)40 $\mu \mathrm{m}$; saco $45(55,2) 82 \mu \mathrm{m}$.

Striapollenites Bharadwaj, 1962

Especie tipo. Striapollenites saccatus Bharadwaj, 1962.

Striapollenites sp. A

(Figura 5U)

Descripción. Grano de polen bisacado, teniado, haploxilonoide a levemente diploxilonoide. Contorno general oval longitudinalmente. Cuerpo central oval longitudinal de márgenes poco discernibles. Capa con 9 a 10 tenias longitudinales subparalelas a oblicuas, mayormente completas (2-3 $\mathrm{m}$ de ancho), las incompletas se acuñan entre las primeras. Cápula de 4/5 del ancho del cuerpo, de forma oval a ahusada longitudinal. Sacos iguales a $1 / 2$ círculo, con ocasionales puentes exinales que los contactan por los extremos. Adherencia proximal ecuatorial con frecuentes pliegues asociados a la zona de adherencia y adherencia distal bilateral.

Dimensiones. (1 ej.). Ancho: total $78 \mu \mathrm{m}$; cuerpo $35 \mu \mathrm{m}$; saco $25 \mu \mathrm{m}$; longitud: cuerpo $77 \mu \mathrm{m}$; saco $90 \mu \mathrm{m}$.

\section{CONSIDERACIONES PALEOECOLÓGICAS}

A partir de la composición de las microfloras (PBSJ 374, 361, 362) de la parte superior de la Formación Andapaico (Tablas 1, 3), es posible señalar que la flora de origen habría estado predominantemente integrada por elementos con requerimientos mesófilos (Gimnospermas s.l., Coníferas, Glossopteridales, Caytoniales, Pteridospermas) con baja participación de elementos de la comunidad higro-hidrófila.

La alta diversidad de granos de polen (a nivel genérico y específico) así como la baja representación de esporas en general, podría explicarse por la desaparición y/o reducción de ambientes con condiciones que facilitaran el desarrollo de floras con requerimientos higro-hidrófilos en las cercanías a las zonas de depositación de la microflora.

\section{CONSIDERACIONES BIOESTRATIGRÁFICAS}

\section{Edad de la microflora}

La Formación Andapaico fue referida al intervalo Cisuraliano-Guadalupiano temprano a partir de su contenido florístico. El registro megaflorístico proviene de la parte inferior de la unidad (Arrondo et al., 1986; Correa et al., 2012) y fue asignado, aunque con dudas, a la Superzona de Ferugliocladus definida para el Pérmico Inferior de la Cuenca Tepuel-Genoa (Archangelsky \& Cúneo, 1984). De forma complementaria, la sección inferior brindó microfloras que permitieron asignar dichos niveles al Cisuraliano temprano (Correa et al., 2012).

Por su parte, los niveles superiores de la Formación Andapaico brindaron las microfloras que aquí son caracterizadas (Tablas 1,2) y han sido referidas por Correa et al., 2012 a la Biozona Lueckisporites-Weylandites (LW), de edad Cisuraliano temprano-Guadalupiano temprano del centro-oeste argentino (Césari \& Gutiérrez, 2001; Césari, 2007). En esta contribución se confirma la pertenencia a la Biozona LW en la microflora analizada a partir de la presencia de un conjunto de taxones, entre ellos: Lueckisporites singhii, L. stenotaeniatus, L. cf. L. angoulaensis, Weylandites lucifer, Corisaccites alutas, Lunatisporites variesectus, Striomonosaccites cicatricosus, Vittatina corrugata, Alisporites parvus, Striatopodocarpites gondwanensis y Vitreisporites $\mathrm{cf}$. V. signatus, entre otros (Tabla 4).

Se propone además, incorporar como nuevos integrantes y componentes exclusivos de la Biozona LW las siguientes especies: Accinctisporites ligatus, Alisporites opii, A. similis, Brazilea sp. A, Corisaccites vanus, Crustaesporites globosus, Cycadopites crassimarginis, C. follicularis, Hamiapollenites erebi, $H$. ruditaeniatus, Limitisporites amazoniensis, $L$. cf. L. delasaucei, Lueckisporites singraulensis, Lunatisporites ovatus, L. cf. L. pellucidus, Protohaploxypinus microcorpus y Striomonosaccites ovatus. Un segundo conjunto de taxones incluirían formas compartidas con la Biozona FS: Calamospora breviradiata, Hamiapollenites bullaeformis, Illinites unicus, Latusipollenites quadrisaccatus, Pakhapites ovatus, Platysaccus cf. P. olivae, Polarisaccites bilateralis, Protohaploxypinus goraiensis, Scheuringipollenites circularis, S. maximus y Tuberisaccites varius. También se incorporan las especies Brevitriletes levis, Caheniasaccites flavatus, Vallatisporites arcuatus, Crucisaccites latisulcatus y Converrucosisporites confluens, presentes en las biozonas FS y DM. Por último, integrarían también la Biozona LW las especies Cyclogranisporites microgranus y Caheniasaccites elongatus, formas presentes en la Biozona DM.

Entre los nuevos elementos que caracterizarían la Biozona LW se destacan algunos que son mencionados por primera vez para el Pérmico de la Argentina y que caracterizan asociaciones pérmicas de otras regiones del Gondwana e incluso de Euroamérica, tales como: Corisaccites vanus (Venkatachala \& Kar, 1968; Bharadwaj et al., 1976; Tiwari \& Tripathi, 1992; Maranhão \& Petri, 1996), Lueckisporites singraulensis (Sinha, 1972), Lunatisporites ovatus (Goubin, 1965; Tiwari \& Vijaya, 1995), Hamiapollenites erebi (Utting, 1994), H. ruditaeniatus (Qu \& Wang, 1986; Ouyang \& Norris, 1990).

Originalmente, la Biozona LW fue referida a la parte final del Pérmico Temprano (no más antiguo que el Artinskiano) (Césari \& Gutiérrez, 2001), posteriormente al Cisuraliano tardío-Guadalupiano temprano (Césari, 2007; Césari et al., 2011), siendo parcialmente equivalente a las biozonas Striatites (Cuenca Chacoparaná en Argentina; Russo et al., 1980) y Lueckisporites virkkiae (Cuenca Paraná en Brasil; Souza \& Marques Toigo, 2005). Las microfloras aquí analizadas provienen de niveles inmediatamente por encima de los depósitos eólicos de la Formación Andapaico, los que estarían reflejando el inicio de un período de aridización en estos sectores del Gondwana (véase Limarino \& Spalletti, 2006) ocurrido durante el Cisuraliano (véase Césari et al., 2011). Es posible sugerir que las mismas podrían representar 
Tabla 4. Distribución bioestratigráfica de las especies identificadas en la asociación de los niveles superiores de la Formación Andapaico. Cuencas del central-oeste argentino: biozonas DM, FS, LW (Gutiérrez \& Césari, 2000; Césari \& Gutiérrez, 2001; Balarino \& Gutiérrez, 2006; Gutiérrez \& Limarino, 2006; Vergel, 2008; Pérez Loinaze et al., 2010; Cisterna et al., 2011; Di Pasquo et al., 2011; Gutiérrez et al., 2010, 2011). Cuenca Chacoparaná (CP): biozonas PL, Plicatipollenites-Lundbladispora; Cr, Cristatisporites; S, Striatites (Archangelsky \& Gamerro, 1979; Russo et al., 1980; Césari et al., 1995; Archangelsky \& Vergel, 1996; Playford \& Dino, 2002). Cuenca Paraná (Brasil): biozonas Ac, Ahrensisporites cristatus; Cm, Crucisaccites monoletus; Vc, Vittatina costabilis: Subzona Pg, Protohaploxypinus goraiensis, Hk, Subzona Hamiapollenites karroensis; Lv, Lueckisporites virkkiae (Souza \& Callegari, 2004; Souza \& Marques-Toigo, 2005; Premaor et al., 2006; Souza, 2006; Félix \& Souza, 2008; Neregato et al., 2008). Cuencas Amazonas y Paranaíba: biozonas St, Spelaeotriletes triangulus; Si, Striomonosaccites incrassatus; lu, Illinites unicus; Sh, Striatosporites heyleri; Rc, Raistrickia cephalata; Vc, Vittatina costabilis (inferior, i; superior, s); Tt, Tornopollenites toreutos (Playford \& Dino, 2000a,b; Dino \& Playford, 2002; Dino et al., 2002; Nascimento et al., 2009; Souza et al., 2010).

Table 4. Biostratigraphic distribution of identified species in the higher levels of the Andapaico Formation. Centra-western basins of Argentina: DM, FS, LW biozones (Césari \& Gutiérrez, 2001; Gutiérrez \& Césari, 2000; Balarino \& Gutiérrez, 2006; Gutiérrez \& Limarino, 2006; Cisterna et al., 2011; Vergel, 2008; Di Pasquo et al., 2011; Gutiérrez et al., 2010; 2011; Pérez Loinaze et al., 2010). Chacoparaná Basin (CP): PL, Plicatipollenites-Lundbladispora; Cr. Cristatisporites; S. Striatites biozones (Archangelsky \& Gamerro, 1979; Russo et al., 1980; Césari et al., 1995; Archangelsky \& Vergel, 1996; Playford \& Dino, 2002. Paraná Basin (Brazil): Ac, Aherensisporites cristatus; Cm, Crucisaccites monoletus; Vc, Vittatina costabilis (Pg, Protohaploxypinus goraiensis; Hk, Hamiapollenites karroensis subzones); Lv, Lueckisporites virkkiae (Souza \& Callegari, 2004; Souza \& Marques-Toigo, 2005; Premaor et al., 2006; Souza, 2006; Felix \& Souza, 2008; Neregato et al., 2008). Amazonas \& Paranaíba Basins: St, Spelaeotriletes triangulatus; Si, Striomonosaccites incressatu; lu, Illinites unicus; Sh, Striatosporites heyleri; Rc, Raistrickia cephalata; Vc, Vittatina costabilis (i, lower, s, upper); Tt, Tornopollenites toreutos (Playford \& Dino, 2000a,b; Dino \& Playford, 2002; Dino et al., 2002; Nascimento et al., 2009; Souza et al., 2010).

\begin{tabular}{|c|c|c|c|c|c|c|c|c|c|c|c|c|c|c|c|c|c|c|c|c|}
\hline \multirow[b]{3}{*}{ Taxones seleccionados } & \multirow{3}{*}{$\begin{array}{c}\text { Cuencas } \\
\text { Biozonas }\end{array}$} & \multicolumn{3}{|c|}{$\begin{array}{l}\text { Centro-oeste } \\
\text { argentino }\end{array}$} & \multicolumn{3}{|c|}{$\begin{array}{l}\text { Chacoparaná } \\
\text { (Argentina) }\end{array}$} & \multicolumn{4}{|c|}{$\begin{array}{l}\text { Paraná } \\
\text { (Brasil) }\end{array}$} & & \multicolumn{8}{|c|}{$\underset{\text { (Brasil) }}{\text { Amazonas \& Paranaíba }}$} \\
\hline & & \multirow{2}{*}{$\mathrm{DM}$} & \multirow{2}{*}{ FS } & \multirow{2}{*}{ LW } & \multirow{2}{*}{ PL } & \multirow{2}{*}{$\mathrm{Cr}$} & \multirow{2}{*}{ S } & \multirow{2}{*}{ Ac } & \multirow{2}{*}{$\mathrm{Cm}$} & \multicolumn{2}{|c|}{$\mathrm{Vc}$} & \multirow{2}{*}{$\mathrm{Lv}$} & \multirow{2}{*}{ St } & \multirow{2}{*}{$\mathrm{Si}$} & & & & $\mathrm{V}$ & & \\
\hline & & & & & & & & & & $\mathrm{Pg}$ & $\mathrm{Hk}$ & & & & $\mathrm{lu}$ & Sh & Rc & $\mathrm{i}$ & $\mathrm{s}$ & $\mathrm{It}$ \\
\hline Cahenisaccites elongatus & & $\mathrm{x}$ & & 0 & & & & $\mathrm{x}$ & $\mathrm{x}$ & $\mathrm{x}$ & $\mathrm{x}$ & $\mathrm{x}$ & & & & & & & & \\
\hline Cyclogranisporites microgranus & & $\mathrm{x}$ & & 0 & & & & & & $\mathrm{x}$ & $\mathrm{x}$ & & & & & & & & & \\
\hline Protohaploxypinus amplus & & $\mathrm{x}$ & $\mathrm{x}$ & $\mathrm{x}$ & & & & $\mathrm{x}$ & $\mathrm{x}$ & $\mathrm{x}$ & & $\mathrm{x}$ & $\mathrm{x}$ & $\mathrm{x}$ & $\mathrm{x}$ & $\mathrm{x}$ & $\mathrm{x}$ & & & \\
\hline Caheniasaccites ovatus & & $\mathrm{x}$ & $\mathrm{x}$ & $\mathrm{x}$ & $\mathrm{x}$ & $\mathrm{x}$ & $\mathrm{x}$ & $\mathrm{x}$ & & $\mathrm{x}$ & $\mathrm{x}$ & & $\mathrm{x}$ & $\mathrm{x}$ & $\mathrm{x}$ & $\mathrm{x}$ & $\mathrm{x}$ & $\mathrm{x}$ & & \\
\hline Cannanoropollis densus & & $\mathrm{x}$ & $\mathrm{x}$ & $\mathrm{x}$ & $\mathrm{x}$ & $\mathrm{x}$ & $\mathrm{x}$ & $\mathrm{x}$ & $\mathrm{x}$ & $\mathrm{x}$ & & & & & $\mathrm{x}$ & & $\mathrm{x}$ & & & \\
\hline Cannanoropollis janakii & & $\mathrm{x}$ & $\mathrm{x}$ & $\mathrm{x}$ & & $\mathrm{x}$ & & $\mathrm{x}$ & $\mathrm{x}$ & $\mathrm{x}$ & & & $\mathrm{x}$ & $\mathrm{x}$ & $\mathrm{x}$ & $\mathrm{x}$ & $\mathrm{x}$ & $\mathrm{x}$ & & \\
\hline Leiotriletes directus & & $\mathrm{x}$ & $\mathrm{x}$ & $\mathrm{x}$ & & & & & & $\mathrm{x}$ & $\mathrm{x}$ & $\mathrm{x}$ & & & & & & & & \\
\hline Scheuringipollenites medius & & $\mathrm{cf}$ & $\mathrm{x}$ & $\mathrm{x}$ & & $\mathrm{x}$ & & & & $\mathrm{x}$ & $\mathrm{x}$ & & & & & & & & & \\
\hline Colpisaccites granulosus & & $\mathrm{x}$ & $\mathrm{x}$ & $\mathrm{x}$ & & $\mathrm{x}$ & $\mathrm{x}$ & & & & & & & & & & & & & \\
\hline Cannanoropollis mehtae & & $\mathrm{x}$ & $\mathrm{x}$ & $\mathrm{x}$ & $\mathrm{x}$ & $\mathrm{x}$ & & & & & & & & & & & & & & \\
\hline Platysaccus queenslandi & & $\mathrm{x}$ & $\mathrm{x}$ & $\mathrm{x}$ & & & & & & & & & & & & & & & & \\
\hline Pteruchipollenites gracilis & & $\mathrm{x}$ & $\mathrm{x}$ & $\mathrm{x}$ & & & & & & & & & & & & & & & & \\
\hline Brevitriletes levis & & $\mathrm{x}$ & $\mathrm{x}$ & 0 & & & & $\mathrm{x}$ & $\mathrm{x}$ & $\mathrm{x}$ & $\mathrm{x}$ & $\mathrm{x}$ & & & & & & & & \\
\hline Caheniasaccites flavatus & & $\mathrm{x}$ & $\mathrm{x}$ & 0 & & & & $\mathrm{x}$ & $\mathrm{x}$ & $\mathrm{x}$ & & $\mathrm{x}$ & & & & & $\mathrm{x}$ & & & \\
\hline Lundbladispora riobonitensis & & $\mathrm{x}$ & $\mathrm{x}$ & $\mathrm{cf}$ & $\mathrm{x}$ & $\mathrm{x}$ & & $\mathrm{x}$ & $\mathrm{x}$ & $\mathrm{x}$ & $\mathrm{x}$ & $\mathrm{x}$ & & & & & & & & \\
\hline Lundbladispora braziliensis & & $\mathrm{x}$ & $\mathrm{x}$ & $\mathrm{cf}$ & $\mathrm{x}(\mathrm{s})$ & $\mathrm{x}$ & & $\mathrm{x}$ & $\mathrm{x}$ & $\mathrm{x}$ & $\mathrm{x}$ & & & & & & & & & \\
\hline Vallatisporites arcuatus & & $\mathrm{x}$ & $\mathrm{x}$ & 0 & $\mathrm{x}$ & $\mathrm{x}$ & & $\mathrm{x}$ & $\mathrm{x}$ & $\mathrm{x}$ & $\mathrm{x}$ & & & & $\mathrm{x}$ & $\mathrm{x}$ & & & & \\
\hline Crucisaccites latisulcatus & & $\mathrm{x}$ & $\mathrm{x}$ & 0 & $\mathrm{x}(\mathrm{s})$ & $\mathrm{x}$ & & $\mathrm{x}$ & & & & & & & $\mathrm{x}$ & $\mathrm{x}$ & & & & \\
\hline Converrucosisporites confluens & & $\mathrm{cf}$ & $\mathrm{x}$ & 0 & $\mathrm{x}(\mathrm{s})$ & $\mathrm{x}$ & $\mathrm{x}(\mathrm{i})$ & & & $\mathrm{x}$ & & & & & & & & & & \\
\hline Potonieisporites lelei & & $\mathrm{x}$ & $\mathrm{x}$ & 0 & & & & & & & & & & & & & $\mathrm{x}$ & & & \\
\hline Barakarites rotatus & & $\mathrm{cf}$ & $\mathrm{x}$ & $\mathrm{cf}$ & & & & & & & & & & & $\mathrm{x}$ & & & & & \\
\hline Retusotriletes diversiformis & & $\mathrm{x}$ & $\mathrm{x}$ & $\mathrm{cf}$ & & $\mathrm{x}$ & & & & & & & & & & & & & & \\
\hline Hamiapollenites fusiformis & & & $\mathrm{x}$ & $\mathrm{x}$ & & & & & & $\mathrm{x}$ & & & & & & & & & $\mathrm{x}$ & $\mathrm{x}$ \\
\hline Lunatisporites variesectus & & & $\mathrm{x}$ & $\mathrm{x}$ & & $\mathrm{x}$ & $\mathrm{x}$ & & & & & $\mathrm{x}$ & & & & & & & & \\
\hline Mabuitasaccites crucistriatus & & & $\mathrm{x}$ & $\mathrm{x}$ & $\mathrm{x}(\mathrm{s})$ & $\mathrm{x}$ & $\mathrm{x}$ & $\mathrm{x}$ & & $\mathrm{x}$ & $\mathrm{x}$ & $\mathrm{x}$ & & $\mathrm{x}$ & $\mathrm{x}$ & & $\mathrm{x}$ & & & \\
\hline Marsupipollenites striatus & & & $\mathrm{x}$ & $\mathrm{x}$ & $\mathrm{x}(\mathrm{s})$ & $\mathrm{x}$ & $\mathrm{x}$ & & & & $\mathrm{cf}$ & $\mathrm{x}$ & & & & & & & & \\
\hline Pakhapites fusus & & & $\mathrm{x}$ & $\mathrm{x}$ & & $\mathrm{x}$ & $\mathrm{x}$ & & & $\mathrm{x}$ & $\mathrm{x}$ & $\mathrm{x}$ & & & & & & $\mathrm{x}$ & $\mathrm{x}$ & $\mathrm{x}$ \\
\hline Vittatina costabilis & & & $\mathrm{x}$ & $\mathrm{x}$ & & $\mathrm{x}$ & $\mathrm{x}$ & & & $\mathrm{x}$ & $\mathrm{x}$ & $\mathrm{x}$ & & & & & & $\mathrm{x}$ & $\mathrm{x}$ & $\mathrm{x}$ \\
\hline Vittatina subsaccata & & & $\mathrm{x}$ & $\mathrm{x}$ & & $\mathrm{x}$ & $\mathrm{x}$ & & & $\mathrm{x}$ & $\mathrm{x}$ & $\mathrm{x}$ & & & & & & $\mathrm{x}$ & $\mathrm{x}$ & $\mathrm{x}$ \\
\hline Striatopodocarpites cancellatus & & & $\mathrm{cf}$ & $\mathrm{x}$ & & $\mathrm{x}$ & $\mathrm{x}$ & & & $\mathrm{x}$ & & $\mathrm{x}$ & & & & & & & & \\
\hline Scheuringipollenites ovatus & & & $\mathrm{x}$ & $\mathrm{x}$ & & & $\mathrm{x}$ & & & $\mathrm{x}$ & $\mathrm{x}$ & $\mathrm{x}$ & & & & & & & & \\
\hline Vittatina vittifera & & & $\mathrm{cf}$ & $\mathrm{x}$ & & & & & & $\mathrm{x}$ & $\mathrm{x}$ & $\mathrm{x}$ & & & & & & $\mathrm{x}$ & $\mathrm{x}$ & $\mathrm{x}$ \\
\hline Alisporites rioclarensis & & & $x$ & $\mathrm{x}$ & & & & & & & & $\mathrm{x}$ & & & & & & & & \\
\hline Vittatina fasciolata & & & $x$ & $\mathrm{x}$ & & & & & & & & $\mathrm{x}$ & & & & & & & & \\
\hline Weylandites magmus & & & $x$ & $\mathrm{x}$ & & & & & & & & & & & & & & & & \\
\hline Alisporites australis & & & $x$ & $x$ & & & & & & & & & & & & & & & & \\
\hline Polarisaccites bilateralis & & & $\mathrm{x}$ & 0 & $\mathrm{x}(\mathrm{s})$ & $\mathrm{x}$ & $\mathrm{x}$ & & & $\mathrm{x}$ & $\mathrm{x}$ & $\mathrm{x}$ & & & & & $\mathrm{x}$ & & & \\
\hline Scheuringipollenites circularis & & & $\mathrm{x}$ & 0 & $\mathrm{x}(\mathrm{s})$ & $\mathrm{x}$ & & & & & & & & & & & & & & \\
\hline Hamiapollenites bullaeformis & & & $\mathrm{cf}$ & 0 & & & & & & & & $\mathrm{x}$ & & & & & & & & \\
\hline
\end{tabular}


Tabla 4. Continua.

\begin{tabular}{|c|c|c|c|c|c|c|c|c|c|c|c|c|c|c|c|c|c|c|c|c|}
\hline \multirow[b]{3}{*}{ Taxones seleccionados } & \multirow{3}{*}{$\begin{array}{c}\text { Cuencas } \\
\text { Biozonas }\end{array}$} & \multicolumn{3}{|c|}{$\begin{array}{l}\text { Centro-oeste } \\
\text { argentino }\end{array}$} & \multicolumn{3}{|c|}{$\begin{array}{l}\text { Chacoparaná } \\
\text { (Argentina) }\end{array}$} & \multicolumn{5}{|c|}{$\begin{array}{l}\text { Paraná } \\
\text { (Brasil) }\end{array}$} & \multicolumn{8}{|c|}{$\begin{array}{c}\text { Amazonas \& Paranaíba } \\
\text { (Brasil) }\end{array}$} \\
\hline & & \multirow{2}{*}{ DM } & \multirow{2}{*}{ FS } & \multirow{2}{*}{ LW } & \multirow{2}{*}{ PL } & \multirow{2}{*}{$\mathrm{Cr}$} & \multirow{2}{*}{$\mathrm{S}$} & \multirow{2}{*}{ Ac } & \multirow{2}{*}{$\mathrm{Cm}$} & \multicolumn{2}{|c|}{$\mathrm{Vc}$} & \multirow{2}{*}{ Lv } & \multirow{2}{*}{ St } & \multirow{2}{*}{$\mathrm{Si}$} & \multirow{2}{*}{$\mathrm{Iu}$} & \multirow{2}{*}{ Sh } & $\mathrm{Rc}$ & $\mathrm{V}$ & & $\mathrm{Tt}$ \\
\hline & & & & & & & & & & $\mathrm{Pg}$ & $\mathrm{Hk}$ & & & & & & $\mathrm{KC}$ & i & $\mathrm{s}$ & 11 \\
\hline Calamospora breviradiata & & & $\mathrm{x}$ & 0 & & & & & & & & & $\mathrm{x}$ & $\mathrm{x}$ & $\mathrm{x}$ & $\mathrm{x}$ & $\mathrm{x}$ & $\mathrm{x}$ & & \\
\hline Illinites unicus & & & $\mathrm{x}$ & 0 & & & & & & $\mathrm{x}$ & & & & $\mathrm{x}$ & $\mathrm{x}$ & $\mathrm{x}$ & $\mathrm{x}$ & & & \\
\hline Pakhapites ovatus & & & $\mathrm{x}$ & 0 & & & & & & $\mathrm{x}$ & $\mathrm{x}$ & & & & & & & $\mathrm{x}$ & $\mathrm{x}$ & $\mathrm{x}$ \\
\hline Latusipollenites quadrisaccatus & & & $\mathrm{x}$ & 0 & & & & & & $\mathrm{x}$ & $\mathrm{x}$ & & & & & & & & & \\
\hline Protohaploxypinus goraiensis & & & $\mathrm{x}$ & 0 & & & & & & $\mathrm{x}$ & & & & & & & & & & \\
\hline Scheuringipollenites maximus & & & $\mathrm{x}$ & 0 & & & & & $\mathrm{x}$ & $\mathrm{x}$ & & & & & & & & & & \\
\hline Platysaccus olivae & & & $\mathrm{x}$ & $\mathrm{cf}$ & & & & & & & & & & & & & & & & \\
\hline Tuberisaccites varius & & & $\mathrm{x}$ & 0 & & & & & & & & & & & & & & & & \\
\hline Corisaccites alutas & & & & $\mathrm{x}$ & & & $\mathrm{x}$ & & & & $\mathrm{x}$ & $\mathrm{x}$ & & & & & & $\mathrm{x}$ & $\mathrm{x}$ & $\mathrm{x}$ \\
\hline Lueckisporites angoulaensis & & & & $\mathrm{x}$ & & & $\mathrm{x}$ & & & & & $\mathrm{x}$ & & & & & & & & \\
\hline Lueckisporites stenotaeniatus & & & & $\mathrm{x}$ & & $\mathrm{x}$ & $\mathrm{x}$ & & & & & $\mathrm{x}$ & & & & & & & & \\
\hline Striomonosaccites cicatricosus & & & & $\mathrm{x}$ & & $\mathrm{x}$ & $\mathrm{x}$ & & & & $\mathrm{x}$ & & & & & & & & & \\
\hline Vittatina corrugata & & & & $\mathrm{x}$ & & $\mathrm{x}$ & $\mathrm{x}$ & & & $\mathrm{x}$ & $\mathrm{x}$ & & & & & & & & & \\
\hline Alisporites parvus & & & & $\mathrm{x}$ & & & & & & & & & & & & & & & & \\
\hline Lueckisporites singhii & & & & $\mathrm{x}$ & & & & & & & & & & & & & & & & \\
\hline Striatopodocarpites gondwanensis & & & & $\mathrm{x}$ & & & & & & & & & & & & & & & & \\
\hline Vitreisporites signatus & & & & $\mathrm{x}$ & & & & & & & & & & & & & & & & \\
\hline Weylandites lucifer & & & & $\mathrm{x}$ & & & & & & $\mathrm{x}$ & $\mathrm{x}$ & $\mathrm{x}$ & & & & & & & & \\
\hline Crustaesporites globosus & & & & 0 & & & & & & & & $\mathrm{x}$ & & & & & & & & \\
\hline Striomonosaccites ovatus & & & & 0 & & & & & & $\mathrm{cf}$ & $\mathrm{cf}$ & $\mathrm{x}$ & $\mathrm{x}$ & $\mathrm{x}$ & $\mathrm{x}$ & $\mathrm{x}$ & $\mathrm{x}$ & $\mathrm{x}$ & & \\
\hline Limitisporites amazoniensis & & & & 0 & & & & & & & & & & & & & $\mathrm{x}$ & $\mathrm{x}$ & & \\
\hline Brazilea sp. A & & & & 0 & & & & & & & & & & & $\mathrm{x}$ & & & $\mathrm{x}$ & & \\
\hline Alisporites opii & & & & 0 & & & & & & & & & & & & & & & & \\
\hline Alisporites similis & & & & 0 & & & & & & & & & & & & & & & & \\
\hline Limitisporites delasaucei & & & & $\mathrm{cf}$ & & & & & & & & $\mathrm{cf}$ & & & & & & & & \\
\hline Corisaccites vanus & & & & 0 & & & & & & & & & & & & & & & & \\
\hline Hamiapollenites erebi & & & & 0 & & & & & & & & & & & & & & & & \\
\hline Hamiapollenites ruditaeniatus & & & & 0 & & & & & & & & & & & & & & & & \\
\hline Lueckisporites singraulensis & & & & 0 & & & & & & & & & & & & & & & & \\
\hline Lunatisporites ovatus & & & & 0 & & & & & & & & $\mathrm{cf}$ & & & & & & & & \\
\hline Lunatisporites pellucidus & & & & $\mathrm{cf}$ & & & & & & & & & & & & & & & & \\
\hline Protohaploxypinus microcorpus & & & & 0 & & & & & & & & & & & & & & & & \\
\hline Cycadopites crassimarginis & & & & 0 & & & & & & & & & & & & & & & & \\
\hline Cycadopites follicularis & & & & 0 & & & & & & & & & & & $\mathrm{x}$ & $\mathrm{x}$ & $\mathrm{x}$ & $\mathrm{x}$ & $\mathrm{x}$ & $\mathrm{x}$ \\
\hline Accinctisporites ligatus & & & & 0 & & & & & & & & & & & & & & & & \\
\hline
\end{tabular}

la parte inferior a media de la Biozona LW, debido a la gran cantidad de elementos comunes (26) que exhibe con la Biozona FS como así también a la afinidad que presentaría tanto con las biozonas Cr y S de la Cuenca Chacoparaná (Tabla 4).

Recientemente se ha dado a conocer una microflora proveniente de la Formación La Veteada (Sistema Famatina), Pérmico de Catamarca (Gutiérrez et al., 2011), la cual ha sido referida a la parte superior de la Biozona LW, en base a la presencia de Convolutispora archangelskyi Playford \& Dino, Lueckisporites nyakapandensis Hart, L. virkkiae (Potonié \& Klaus) Klaus, Staurosaccites cordubensis Archangelsky \& Gamerro y Striatoabieites anaverrucosus Archangelsky \& Gamerro, entre otras; formas que no han sido halladas en la asociación aquí estudiada. Además, en dicha microflora se hallaron Grebespora concentrica Jansonius, Klausipollenites spp., Minutosaccus spp., etc., que caracterizan microfloras del Pérmico Medio-Tardío y Triásico (para registros previos véase Gutiérrez et al., 2011). La comparación entre los componentes de las asociaciones de Andapaico y La Veteada permitiría sugerir para la microflora de Andapaico una edad más antigua respecto de la segunda.

\section{Comparaciones con cuencas sudamericanas}

La asociación aquí estudiada fue comparada con los elementos que conforman las biozonas propuestas para Chacoparaná (Russo et al., 1980; Archangelsky \& Vergel, 1996; Tabla 4). Si bien existe una mayor correspondencia en cuanto a la cantidad de especies en común con la Biozona Cristatisporites - Cr- (24 especies) que con la Biozona Striatites - S- (18 especies), la ausencia de los principales taxones que caracterizan a la Biozona $\mathrm{Cr}$ (alta diversidad específica del género Cristatisporites, predominio del polen monosacado, esporas triletes apiculadas y cingulizonadas), así como la presencia de Corisaccites alutas, Lueckisporites cf. L. angoulaensis y Scheuringipollenites ovatus, permitirían plantear una mayor afinidad con la Biozona S. 
Con respecto a la biozonación propuesta para la Cuenca Paraná (Souza \& Marques-Toigo, 2005; Souza, 2006; véase Tabla 4), se pueden observar similitudes tanto con la Biozona Vitatina costabilis -Vc- debido a la abundancia de especies de los géneros Vittatina, Protohaploxypinus e Ilinites, como así también con la Biozona Lueckisporites vikkiae -Lv. Aunque no se halle $L$. virkkiae en la microflora de Formación Andapaico, sí abundan otros elementos característicos de Lv como Weylandites lucifer, y especies pertenecientes a los géneros Protohaploxypinus, Lueckisporites, Lunatisporites y Striatipodocarpites. Con respecto a las biozonas propuestas para la Cuenca Amazonas (Playford \& Dino, 2000a,b), existe un elevado número de coincidencias entre los elementos aquí estudiados con los que conforman dichas biozonas (Tabla 4). Teniendo en cuenta el bajo número de especies que las definen, se infiere que la asociación aquí estudiada se asemeja a la Biozona Vittatina costabilis -Vc- por la presencia de Vittatina costabilis, $V$. subsaccata, $V$. vittifera, Corisaccites alutas, Hamiapollenites fusiformis y Pakhapites fusus. Si bien estas especies también están presentes en la Biozona Tornopollenites toreutus -Tt-, la ausencia de los elementos diagnósticos (Laevigatosporites minor, Thymospora obscura, Verrucosisporites insuetus, Tornopollenites toreutos) no permiten una mayor grado de correlación.

Estas afinidades poco marcadas con las biozonas definidas, probablemente se deban a la combinación de varios factores, entre ellos mencionaremos: (i) los diferentes ambientes en que se depositaron las sedimentitas portadoras de las microfloras en cuestión: áreas de sedimentación continental (Cuencas Chacoparaná, Paraná y Amazonas) versus sedimentación continental a litoral (próxima a las cuencas marinas del margen occidental del Gondwana para el sector occidental de la Cuenca Paganzo); y (ii) baja densidad de datos disponibles: a diferencia de los numerosos hallazgos de subsuelo en las cuencas orientales (Chacoparaná-Paraná-Amazonas), en el margen occidental del Gondwana los registros de superficie son escasos (véase Césari \& Gutiérrez, 2001; Pérez Loinaze et al., 2010; Gutiérrez et al., 2011, y este trabajo).

\section{CONCLUSIONES}

El análisis de tres microfloras provenientes de la parte alta de la Formación Andapaico, ha permitido identificar una asociación referida a la Biozona Lueckisporites-Weylandites (LW), cuya edad sería Cisuraliana media-Guadalupiana temprana a partir de la presencia de: Alisporites parvus, Vitreisporites cf. V. signatus, Striomonosaccites cicatricosus, Corisaccites alutas, Lueckisporites cf. L. angoulaensis, L. stenotaeniatus, L. singhii, Lunatisporites variesectus, Striatopodocarpites gondwanensis, Vittatina corrugata y Weylandites lucifer, como así también por ubicarse las microfloras inmediatamente por encima de las facies eólicas. Dichas facies reflejarían el inicio de una aridización registrada para el Cisuraliano, por lo que es probable que representen los sectores inferiores y/o medios de la Biozona LW.

La edad de la parte alta de la Formación Andapaico se afirma a partir del alto grado de similitud con los elementos que conforman las biozonas Cr y S (Cuenca Chacoparaná), en especial con la Biozona $\mathrm{S}$, dado por la abundancia específica de granos de polen estriados y plicados, y la baja representación de esporas apiculadas y cingulizonadas, granos de polen monosacados, etc. Por su parte, con la Cuenca Paraná presenta elementos afines a sus dos biozonas pérmicas (Vc y Lv), y con la Cuenca Amazonas, encuentra un mayor grado de similitud con la Biozona Vc. La edad también fue ajustada a partir de la comparación con la microflora de La Veteada, Catamarca, asignada a la parte superior de la Biozona LW, y no se correspondería con ésta por no hallarse elementos tales como: Convolutispora archangelskyi, Staurosaccites cordubensis y Striatoabieites anaverrucosus, entre otros. Se propone además incorporar como nuevos elementos de la Biozona LW los siguientes taxones: Accinctisporites ligatus, Alisporites opii, A. similis, Brazilea sp. A, Brevitriletes levis, Caheniasaccites flavatus, C. elongatus, Calamospora breviradiata, Ciclogranisporites microgranus, Converrucosisporites confluens, Corisaccites vanus, Crucisaccites latisulcatus, Crustaesporites globosus, Cycadopites crassimarginis y C. follicularis, Hamiapollenites bullaeformis, $H$. erebi, H. ruditaeniatus, Illinites unicus, Latusipollenites quadrisaccatus, Limitisporites amazoniensis, Lueckisporites singraulensis, Lunatisporites ovatus, Pakhapites ovatus, Polarisaccites bilateralis, Potonieisporites lelei, Protohaploxypinus microcorpus, P. goraiensis, Scheuringipollenites circularis, S. maximus, Striomonosaccites ovatus, Tuberisaccites varius, Vallatisporites arcuatus. A partir del análisis de los grupos parentales que reflejan éstas microfloras, se puede inferir una flora con requerimientos mesófilos con una baja participación de elementos de la comunidad higro-hidrófila que reflejen condiciones húmedas próximas a las zonas de depositación.

\section{AGRADECIMIENTOS}

Los autores desean expresar su agradecimiento a B. Cariglino y E.P. Coturel, por la invalorable ayuda prestada en las tareas de campo. También agradecer especialmente a los revisores, M. di Pasquo y R. Dino por las observaciones y sugerencias, como así también a M.J. Garcia por las correcciones realizadas, que contribuyeron a mejorar ampliamente este trabajo. Estos estudios se llevaron a cabo como parte de proyectos financiados por la Agencia Nacional de Promoción Científica y Técnica (ANPCYT-PICT 32693 y PRESTAMO BID-PICT 2010-2196) y el por CONICET (PIP 0705).

\section{REFERENCIAS}

Archangelsky, S. 1971. Las tafofloras del Sistema Paganzo en la República Argentina. Anais da Academia Brasileira de Ciências, 43(supl.):67-88.

Archangelsky, S. \& Azcuy, C.L. 1985. Carboniferous paleobotany and palynology in Argentina. In: CONGRES DE STRATIGRAPHIE ET DE GEOLOGIE DU CARBONIFERE, 10, 1983. Compte Rendu, Madrid, v. 4, p. 267-280.

Archangelsky, S. \& Cúneo, N. R. 1984. Zonación del Pérmico continental de Argentina sobre la base de sus plantas fósiles. In: 
CONGRESO LATINOAMERICANO DE PALEONTOLOGÍA, 3, 1984. Memorias, México, p. 143-154.

Archangelsky, S. \& Cúneo, R. 1991. The Neopaleozoic floristic sucession from northwestern Argentina. A new perspective. In: H. Ulbrech \& A.C. Rocha Campos (eds.) INTERNATIONAL GONDWANA SYMPOSIUM, 7, 1991. Resumos expandidos, São Paulo, p. 469-481.

Archangelsky, S. \& Gamerro, J.C. 1979. Palinología del Paleozoico Superior en el subsuelo de la Cuenca Chacoparanense, República Argentina. I. Estudio sistemático de los palinomorfos de tres perforaciones de la Provincia de Córdoba. Revista Española de Micropaleontología, 11:417-478.

Archangelsky, S \& Vergel, M. M. 1996. Paleontología, bioestratigrafía y paleoecología. In: S. Archangelsky (ed.) El sistema Pérmico en la República Argentina y en la República Oriental del Uruguay, Academia Nacional de Ciencias de Córdoba, p. 40-44.

Arrondo, A. G.; Morel, E.; Cuerda, A. \& Ganuza, D. 1986. Estratos plantíferos del Carbónico de Bachongo, precordillera de San Juan. In: CONGRESO ARGENTINO DE PALEONTOLOGÍA Y BIOESTRATIGRAFÍA, 4, 1986. Actas, Mendoza, p. 237-247.

Arrondo, O.; Morel, E.; Cuerda, A. \& Ganuza, D. 1990. Los depósitos carboníferos del Río Santa Rosa-Bachongo. Late Paleozoic of South America, Proyecto $n^{\circ} 211$, IUGS-UNESCO. Encuentro Anual del Grupo Argentino de Trabajo, p. 5-8 (Comunicaciones).

Azcuy, C.L.; Carrizo, H.A. \& Caminos, R. 2000. Capítulo 12: Carbonífero y Pérmico de las Sierras Pampeanas, Famatina, Precordillera, Cordillera Frontal y Bloque San Rafael. Anales Instituto de Geología y Recursos Minerales, 26:261-317.

Azcuy, C.L.; di Pasquo, M. \& Valdivia Ampuero, H. 2002. Late Carboniferous miospores from the Tarma Formation, Pongo de Mainique, Peru. Review of Palaeobotany and Palynology, 118:1-28. doi:10.1016/S0034-6667(01)00105-1

Backhouse, J. 1991. Permian palynostratigraphy of the Collie Basin, Western Australia. Review of Palaeobotany and Palynology, 67:237-314. doi:10.1016/0034-6667(91)90046-6

Balarino, M.L. \& Gutiérrez, P.R. 2006. Palinología de la Formación Tasa Cuna (Pérmico Inferior), Córdoba, Argentina: sistemática y consideraciones bioestratigráficas. Ameghiniana, 43:437-460.

Balme, B.E. 1957. Spores and pollen grains from the Mesozoic of Western Australia. C.S.I.R.O. Australian Coal Research Section T.C., 25:1-48.

Balme, B.E. 1970. Palynology of Permian and Triassic strata in the Salt Range and Surghar Range, West Pakistan. In: B. Kummel \& C. Teichert (eds.) Stratigraphic boundary problems: Permian and Triassic of West Pakistan, University of Kansas, Special Publication, 4:305-453.

Balme, B.E. 1995. Fossil in situ spores and pollen grains: an annotated catalogue. Review of Palaeobotany and Palynology, 87:81-323. doi:10.1016/0034-6667(95)93235- $X$

Balme, B.E. \& Hennelly, P.F. 1956. Trilete sporomorphs from Australian Permian sediments. Australian Journal of Botany, 4:240-260. doi:10.1071/BT9560240

Balme, B.E. \& Playford, G. 1967. Late Permian plant microfossils from the Prince Charles Mountains, Antarctica. Revue de Micropaléntologie, 10:179-192.

Bharadwaj, D.C. 1962. The miospore genera in the coals of Raniganj Stage (Upper Permian), India. The Palaeobotanist, 9:68-106.

Bharadwaj, D. C. \& Srivastava, S. C. 1969. Some new miospores from Barakar Stage, lower Gondwana, India. The Paleobotanist, 17:220-229.

Bharadwaj, D.C. \& Tiwari, R. S. 1964. On two monosaccate genera from Barakar Stage, India. The Palaeobotanist, 12:139-146.
Bharadwaj, D.C.; Kar, R.K. \& Navale, G.K.B. 1976. Palynostratigraphy of the Lower Gondwana deposits in Paraná and Maranhão basins, Brazil. Biological Memoirs, 1:53-108.

Bose, M.N. \& Kar, R.K. 1966. Paleozoic sporae dispersae from Congo. I-Kindu Kalima and Walekale Regions. Annales de Musée Royal de l'Afrique Centrale, 53:1-238.

Bose, M.N. \& Maheshwari, H.K. 1968. Paleozoic sporae dispersae from Congo. VIII. Area in he vicinity of Lake Tanganyke, South Alberville. Annales de Musee Royal de l'Afrique Centrale, Serie 8, Sciences Géologiques, 60:1-116.

Bracaccini, O. 1950. Investigaciones tectónicas en la Precordillera Sanjuanina. Boletin de Informaciones Petroleras, 301(Reimpresión):1-36.

Césari, S.N. 2007. Palynological biozones and radiometric data at the Carboniferous-Permian boundary in Western Gondwana. Gondwana Research, 11:529-536. doi:10.1016/j.gr.2006.07.002

Césari, S.N.; Archangelsky, S. \& de Seoane, L.V. 1995. Palinología del Paleozoico Superior de la Perforación Las Mochas, provincia de Santa Fe, Argentina. Ameghiniana, 32:73-106.

Césari, S.N. \& Gutiérrez, P.R. 2001. Palynostratigraphy of Upper Paleozoic sequences in Central-Western Argentina. Palynology, 24:113-146. doi:10.2113/0240113

Césari, S.N.; Limarino, C.O. \& Gulbranson, E.L. 2011. An upper Paleozoic bio-chronostratigraphic scheme for the Western margin of Gondwana. Earth Science Reviews, 106:149-160. doi:10.1016/j.earsci.rev.2011.01.012

Cisterna, G.A.; Andrea F., Sterren, A.F. \& Gutiérrez, P.R. 2011. The Carboniferous-Permian boundary in the South American central western Argentinean basins: paleontological evidences. Andean Geology, 38:349-370. doi:10.5027/andgeoV38n2-a06

Clarke, R.F.A. 1965. British Permian saccate and monosulcate miospores. Palaeontology, 8:322-354.

Coca, M. \& Bercowski, F. 1994. Facies eólicas en el Neopaleozoico de Bachongo, Precordillera Central, San Juan. In: REUNIÓN ARGENTINA DE SEDIMENTOLOGÍA, 5, 1994. Actas, San Luis, p. 71-76.

Correa, G.A.; Carrevedo, M.L. \& Gutiérrez, P.R. 2012. Paleoambiente y paleontología de la Formación Andapaico (Paleozoico Superior, Precordillera Central, Argentina). Andean Geology, 39:22-52. doi:10.5027/andgeo02

Daugherty, L.H. 1941. The Upper Triassic Flora of Arizona. Carnegie Institute of Washington, 526:1-108.

de Jersey, N.J. 1962. Triassic spores and pollen grains from the Ipswich Coalfield. Geologycal Survey of Queensland, 307:1-20.

de Jersey, N.J. 1964. Triassic spores and pollen grains from the Bundamba Group. Geologycal Survey of Queensland, 313:1-15.

Dias-Fabrício, M. E. 1981. Palinologia da Formação Rio Bonito na Área de Gravataí-Morungava, Rio Grande do Sul. Pesquisas, 14:69-130.

Dino, R.; Antonioli, L. \& Braz, S.M. 2002. Palynologycal data from the Trisidela Member of Upper Pedra de Fogo Formation ("Upper Permian") of the Paranaíba Basin, Northeastern Brazil. Revista Brasileira de Paleontologia, 3:24-35.

Dino, R. \& Playford, G. 2002. Stratigraphic and palaeoenvironmental significans of Pennsylvanian (Upper Carboniferous) palynoflora from the Piauí Formation, Paranaíba Basin, northeastern Brazil. Paleontological Research, 6:23-40.

Di Pasquo M.; Vergel, M.M. \& Azcuy, C.L. 2011. Pennsylvanian and Cisuralian palynofloras from the Los Sauces area, La Rioja Province, Argentina: chronological and paleoecological significance. International Journal Coal of Geology, 83:276-291. doi:10.1016/j.coal.2010.01.006

Felix, C.M. \& Souza, P.A. 2008. Análise palinotaxonômica e 
bioestratigráfica da Subturma Monosaccites na Bacia do Paraná, Brasil. II: gêneros Costatascyclus, Circumplicatipollis, Caheniasaccites, Stellapollenites e Divarisaccus. Revista Pesquisas em Geociências, 35:57-69.

Foster, C.B. 1975. Permian plant microfossils from the Blair Athol Coal Measures, central Queensland, Australia. Palaeontographica Abteilung B, 154:121-171.

Foster, C.B. 1979. Permian plant microfossils of the Blair Athol Coal Measures, Baralaba Coal Measures, and basal Rewan Formation of Queensland. Geologycal Survey of Queensland, 372:1-244.

Frakes, L.A. 1979. Climates throughout Geologic Time. Elsevier, $310 \mathrm{p}$.

García, G.B. 1996. Palinología de la Formación El Imperial, Paleozoico Superior, Cuenca San Rafael, República Argentina. Parte II: granos de polen, incertae sedis, acritarcas. Ameghiniana, 33:7-33.

González Bonorino, G. 1991. Evolución comparada de la precordillera de San Juan en el Paleozoico temprano y del margen atlántico de Norteamérica en el Mesozoico-Cenozoico. Revista de la Asociación Geológica Argentina, 46:10-19.

Gutiérrez, P.R. 1993. Palinología de la Formación Agua Colorada (Carbonífero Superior), Sierra de Famatina, Provincia de La Rioja, Argentina. I. Granos de polen. Ameghiniana, 30:163-212.

Gutiérrez, P.R. \& Césari, S.N. 2000. Palinología de la Formación Bajo de Véliz (Pérmico Inferior), San Luis, Argentina: revisión sistemática y consideraciones estratigráficas. Ameghiniana, 37:439-462.

Gutiérrez, P.R.; Correa, G.A. \& Carrevedo, M.L. 2010. Primer registro de palinomorfos de edad pérmica en la Formación Río Francia (Paleozoico Superior, San Juan, Argentina). Revista del Museo Argentino de Ciencias Naturales, 12:203-216.

Gutiérrez, P.R.; Ganuza, D.G.; Morel, E. \& Arrondo, O.G. 1992. Los géneros Cordaicarpus Geinitz, Cornucarpus Arber y Samaropsis Goeppert (Semillas platispérmicas) en el Neopaleozoico Argentino. Ameghiniana, 29:49-68.

Gutiérrez, P.R. \& Limarino, C.O. 2006. El perfil del sinclinal del Rincón Blanco (noroeste de La Rioja): el límite CarboníferoPérmico en el noroeste Argentina. Ameghiniana, 43:687-703.

Gutiérrez, P.R.; Zavattieri, A.M.; Ezpeleta, M. \& Astini, R.A. 2011. Palynology of the La Veteada Formation (Permian) at the Sierra de Narváez, Catamarca Province, Argentina. Ameghiniana, 48:154-176. doi:10.5710/AMGH.v48i2(312)

Harrington, J.H. 1971. Descripción geológica de la Hoja 22c, "Ramblon", Provincias de Mendoza y San Juan. Dirección Nacional de Geología y Minería, 87 p. (Boletín 114).

Hart, G.F. 1964. A review of the classification and distribution of Permian miospores: Disaccate Striatiti. In: CONGRES INTERNATIONAL DU STRATIGRAPHIE ET DE GEOLOGIE DU CARBONIFERE, 5, 1963. Compte Rendu, Paris, v. 3, p. 1171-1199.

Hart, G.F. 1965. Microflora from the Ketewaka - Mchuchuma Coalfield, Tanganyika. Bulletin Geological Survey Tanganyika, 36:1-27.

Helby, R. 1973. Review of Late Permian and Triassic palynology of New South Wales. Geological Society of Australia, special publication, 4:141-155.

Jansonius, J. 1962. Palynology of Permian and Triassic sediments, Peace river area, western Canadá. Palaeontographica Abteilung $B, \mathbf{1 1 0}: 35-98$.

Jansonius, J. \& Hills, L.V. 1976. Genera file of fossil spores and pollen. Department of Geology, University of Calgary, Canadá (Special Publication).
Jardiné, S. 1974. Microflores des formations du Gabon attribuées au Karroo. Review of Palaeobotany and Palynology, 17:75-112. doi:10.1016/0034-6667(74)90093-1

Klaus, W. 1963. Sporen aus dem südalpinen Perm. Jahrbuch der Geologischen Bundensanstalt, 106:229-361.

Kosanke, R.M. 1950. Pennsylvanian spores of Illinois and their use in correlation. Illinois State Geological Survival Bulletin, 74, 128 p.

Kräusel, R. \& Leschik, G. 1955. Die Keuperflora von Neuewelt bei Basel, II. Die Iso-und Mikrosporen. Schweizeirische Paläontologische Abhandlungen, 72:1-10.

Lakhanpal, R.N.; Sah, S.C.D. \& Dube, S.N. 1960. Further observations on plant microfossils from a carbonaceous shale (Krols) near Naini Tal, with a discussion on the age of the beds. The Palaeobotanist, 7:111-120.

Lele, K.M. \& Maithy, P.K. 1964. An unusual monosaccate spore from the Karharbari Stage, Giridh Coalfield, India. The Palaeobotanist, 12:307-312.

Lele, K.M. \& Makada, R. 1972. Studies in the Talchir Flora of India - 7. Palynology of the Talchir Formation in the Jayanti Coalfield, Bihar. Geophytology, 2:41-73.

Leschik, G.F. 1955. Die Keuperflora von Neuewelt bei Basel. II. Die Iso-und Mikrosporen. Schweizerische Paläontologische Abhandlungen, 72:1-70.

Leschik, G.F. 1956. Sporen aus dem Salzton des Zechsteins von Neuhof (Bei Fulda). Palaeontographica Abteilung B, 100:122142.

Limarino, C.O. \& Spalletti, L.A. 2006. Paleogeography of the upper Paleozoic basins of southern South America: An overview. Journal of South American Earth Sciences, 22:134-155. doi: 10.1016/j.jsames.2006.09.011

Limarino, C.; Gutiérrez, P.R.; López-Gamundí, O.R.; Fauqué, L. \& Lech, R.R. 1996. Capítulo VIII. Cuencas Río Blanco y CalingastaUspallata. In: S. Archangelsky (ed.) El Sistema Pérmico en la República Argentina y en la República Oriental del Uruguay. Academia Nacional de Ciencias, p.141-154.

Limarino, C.O.; Césari, S.; Net, L.; Marenssi, S.; Gutiérrez, P.R. \& Tripaldi, A. 2002. The Upper Carboniferous postglacial transgression in the Paganzo and Río Blanco Basins (northwestern Argentina): facies and stratigraphic significance. Journal of South American Earth Sciences, 15:445-460. doi:10.1016/ S0895-9811(02)00048-2

Limarino, C.O.; Tripaldi, A.; Marenssi, S. \& Fauque, L. 2006. Tectonic, sea-level, and climatic controls on Late Paleozoic sedimentation in the western basins of Argentina. Journal of South American Earth Sciences, 22:205-226. doi:10.1016/j. jsames.2006.09.009

Maheshwari, H.K. 1967. Studies in the Glossopteris flora of India. 29. Miospora assemblage from the Lower Gondwana Exposures along Bansloi river in Rajmahal Hills, Bihar. The Palaeobotanist, 15:258-280.

Maheshwari, H.K. \& Banerjee, J. 1975. Lower Triassic palynomorphs from the Maitur Formation, West Bengal, India. Palaeontographica Abteilung B, 152:149-190.

Maranhão, M.d.S.A.S. \& Petri, S. 1996. Novas ocorrências de fósseis nas formações Corumbatai e Estrada Nova do Estado de São Paulo e considerações preliminares sobre seus significados paleontológico e bioestratigráfico. Boletim do Instituto de Geociências da Universidade de São Paulo, 17:33-54.

Marques-Toigo, M. 1974. Some new species of spores and pollen of Lower Permian age from the San Gregorio Formation in Uruguay. Anais da Academia Brasileira de Ciências, 46:602616. 
Marques-Toigo, M. \& Picarelli, A.T. 1985. On the morphology and botanical affinities of Lundbladispora Balme, 1963, in the Permian of the Paraná Basin, Brazil. Boletim do Instituto de Geociencias da Universidade de São Paulo, 15:24-52.

Menéndez, C.A. 1971. Estudio palinológico del Pérmico de Bajo de Vélez, provincia de San Luis. Revista del Museo Argentino de Ciencias Naturales "Bernardino Rivadavia” e Instituto Nacional de Investigación de las Ciencias Naturales, Paleontología, 1:263-306.

Menéndez, C.A. 1976. Contenido palinológico de estratos pérmicos con "Mesosaurus" de Río Claro, São Paulo, Brasil. Revista del Museo Argentino de Ciencias Naturales "Bernardino Rivadavia” e Instituto Nacional de Investigación de las Ciencias Naturales, Paleontología, 2:1-30.

Morbey, S.J. 1975. The palynostratigraphy of the Rhaetian Stage, Upper Triassic in the Kendelbachgraben, Austria. Palaeontographica Abteilung B, 152:1-75.

Nascimento, S.; Smaniotto, L.P.; Souza, P.A.; Lemos, V.B. \& Scomazzon, A.K. 2009. Biochronostratigraphy (conodonts and palynology) from a selected strata of the Itaituba Formation (Pennsylvanian of the Amazonas Basin) at Itaituba, Pará State, Brazil. Revista Pesquisas em Geociências, 36:37-47.

Neregato, R.; Souza, P.A. \& Rohn, R. 2008. Registros palinológicos inéditos nas Formações Teresina e Rio do Rasto (Permiano, Grupo Passa Dois, Bacia do Paraná). Implicações biocronoestratigráficas e paleoambientais. Revista Pesquisas em Geociências, 35:9-21.

Olivares Milla, I.G. 2002. Estudio geológico estructural en el extremo sur de precordillera central (Cerro Bachongo), departamento de Sarmiento, provincia de San Juan. Universidad Nacional de San Juan, Tesis Doctoral, 75 p.

Ottone, E.G.; Álvarez, P.P. \& Benoit, S.V. 1992. Late Triassic plant microfossils from the Rancho de Lata Formation, Main Cordillera, Argentina. Micropaleontology, 38:261-278.

Ottone, E.G. \& Azcuy, C.L. 1988. Circumplicatipollis, nuevo género de monosacado del Paleozoico superior de Argentina. Revista Española de Micropaleontología, 20:245-249.

Ouyang, S. \& Norris, G. 1990. Earliest Triassic (Induan) spores y pollen from the Junggar Basin, Xinjiang, northwestern China. Review of Palaeobotany and Palynology, 106:1-56. doi: 10.1016/S0034-6667(98)00078-5

Pérez Loinaze, V.; Ciccioli, P.L.; Limarino, C.O. \& Césari, S.N. 2010. Hallazgo de palinofloras pérmicas en la Precordillera de Mendoza: su implicancia estratigráfica. Ameghiniana, 47:263-269.

Playford, G. \& Dettmann, M.E. 1996. Spores. In: J. Jansonius \& D.C. McGregor (eds.) Palynology: Principles and Applications. American Associations of Stratigraphic Palynologists Foundation, 1:227-260.

Playford, G. \& Dino, R. 2000a. Palynoestratigraphy of upper Palaeozoic strata (Trapajós Group), Amazonas Basin, Brazil: Part One. Palaeontographica Abteilung B, 255:1-46.

Playford, G. \& Dino, R. 2000b. Palynoestratigraphy of upper Palaeozoic strata (Trapajós Group), Amazonas Basin, Brazil: Part Two. Palaeontographica Abteilung B, 255:87-145.

Playford, G. \& Dino, R. 2002. Permian palynofloral assemblages of the Chaco-Paraná Basin, Argentina: systematics and stratigraphic significance. Revista Española de Micropaleontología, 34:235288.

Potonié, R. \& Klaus, W. 1954. Einige Sporegattungen des alpinen Salzgebirges. Geologisches Jahrbuch, 68:517-546.

Potonié, R. \& Kremp, G. 1954. Die Gattungen der Paläozoischen sporae dispersae und ihre stratigraphie. Beihefte zum Geologisches Jahrbuch, 69:111-194.
Potonié, R. \& Sah, S.C.D. 1960. Sporae dispersae of the Lignites from Cannanore Beach on the Malbar Coast of India. The Palaeobotanist, 7:121-135.

Premaor, E.; Fischer, T.V. \& Souza, P.A. 2006. Palinologia da Formação Irati (Permiano Inferior da Bacia do Paraná) em Montividiu, Goias, Brasil. Revista del Museo Argentino de Ciencias Naturales, 8:221-230.

Qu, L.F. \& Wang, Z. 1986. Triassic spore and pollen. In: Geological Society Publishing House (ed.) Geological Memory Series 2, 3 , Beijing, 113-173 p.

Ramos, V. A. \& Vujovich, G. I. 2000. Hoja Geológica 3169-IV, San Juan, Provincia de San Juan. Subsecretaría de Minería Nación, Servicio Geológico Minero Argentino, 82 p. (Boletín 243).

Russo, A.; Archangelsky, S. \& Gamerro, J.C. 1980. Los depósitos suprapaleozoicos en el subsuelo de la llanura ChacoPampeana, Argentina. In: CONGRESO ARGENTINO DE PALEONTOLOGÍA Y BIOESTRATIGRAFÍA, $1 \mathrm{y}$ CONGRESO LATINOAMERICANO DE PALEONTOLOGÍA, 2, 1978. Actas 4, Buenos Aires, p. 157-173.

Salfity, J.A. \& Gorustovich, J.A. 1983. Paleogeografía de la cuenca del Grupo Paganzo (Paleozoico superior). Revista de la Asociación Geológica Argentina, 38:437-453.

Samoilovich, S.R. 1953. Pollen and spores from the Permian deposits of the Cherdyn' and Akt'ubinsk areas, Cis-Urals. Travau del' Institut Petrographyque de l'Academie des Sciences de l'URSS, 75:5-57.

Schaarschmidt, F. 1963. Sporen und Hystrichosphaerideen aus dem Zechstein von Bödingen in der Wetterau. Palaeontographica Abteilung B, 113:38-91.

Sinha, V. 1972. Sporae dispersae from Jhingurdah Seam, Singrauli Coalfield (M.P.), India. The Palaeobotanist, 19:175-201.

Souza, P.A. 2006. Late Carboniferous palynostratigraphy of the Itararé Subgroup, northeastern Paraná Basin, Brazil. Review of Palaeobotany and Palynology, 138:9-29. doi:10.1016/j. revpalbo.2005.09.004

Souza, P.A. \& Callegari, L.M. 2004. An Early Permian Palynoflora from the Itararé Subgroup, Paraná Basin, Brazil. Revista Española de Micropaleontología, 36:439-450.

Souza, P.A. \& Marques-Toigo, M. 2005. Progress on the palynostratigraphy of the Permian Strata in Rio Grande do Sul State, Parana Basin, Brazil. Anais da Academia Brasileira de Ciências, 77:353-365.

Souza, P.A.; Matzembacher, L.T.; Abelha, M. \& Borghi, L. 2010. Palinologia da Formação Piauí, Pensilvaniano da Bacia do Parnaíba: biocronoestratigrafia de intervalo selecionado do Poço 1-UN-09-PI (Caxias, MA, Brasil) Revista Brasileira de Paleontologia, 13:57-66. doi:10.4072/rbp.2010.1.07

Tiwari, R.S. 1973. Scheuringipollenites, a new name for the Gondwana palynomorphs so far assigned to "Sulcatisporites Leschik 1955”. Senckenbergiana Lethaea, 54:105-117.

Tiwari, R.S. \& Tripathi, A. 1992. Marker assemblage zones of spore and pollen species through Gondwana Palaeozoic and Mesozoic sequence in India. The Palaeobotanist, 40:194-236.

Tiwari, R.S. \& Vijaya 1995. Differential morphographic identity of Gondwanic palynomorphs. The Palaeobotanist, 44:62-115.

Tschudy, R.H. \& Kosanke, R.M. 1966. Early Permian vesiculate pollen from Texas, USA. The Palaeobotanist, 1:59-72.

Utting, J. 1994. Palynostratigraphy of Permian and Lower Triassic rocks, Sverdrup Basin, Canadian Artic Archipielago. Geological Survey of Canada, 478:1-107.

Venkatachala, B.S. \& Kar, R.K. 1966. Corisaccites gen. nov., a new saccate pollen genus from the Permian of Salt Range, West Pakistan. The Palaeobotanist, 15:107-109. 
Venkatachala, B.S. \& Kar, R.K. 1968. Palynology of the Karanpura sedimentary Basin, Bihar, India-1. Barakar Stage at Badam. The Palaeobotanist, 16:56-90.

Vergel, M.M. 2008. Palynology of late Palaeozoic sediments (Tupe Formation) at La Herradura Creek, San Juan province, Argentina. Alcheringa, 32:339-352. doi:10.1080/03115510802417075

Wilson, L.R. 1962. Permian plant microfossils from the Flowerpot Formation, Greer Country, Oklahoma. Oklahoma Geological Survey, 49:1-50.

Wilson, L.R. \& Webster, R.M. 1946. Plant microfossils from a
Fort Union coal of Montana. American Journal of Botany, 33:271-278.

Wood, G.D.; Gabriel, A.M. \& Lawson, J.C. 1996. Palynological techniquesprocessing and microscopy. In: J. Jansonius \& D.C. McGregor (eds.) Palynology: principles and applications, American Associations of Stratigraphic Palynologists Foundation, p. 29-50.

Ybert, J.P. \& Marques-Toigo, M. 1971. Polarisaccites nov. gen. Pollen et Spores, 12:469-481.

Received in May, 2011; accepted in July, 2012.

\section{Apéndice/Appendix \\ Esporas trilete \\ Apiculatasporites sp.}

Brevitriletes levis (Balme \& Hennelly) Bharadwaj \& Srivastava, 1969 (Figura 3A)

Calamospora breviradiata Kosanke, 1950 (Figura 3B)

Converrucosisporites confluens (Archangelsky \& Gamerro) Playford \& Dino, 2002 (Figura 3C)

Cyclogranisporites microgranus (Balme \& Hennelly) Playford \& Dino, 2002 (Figura 3D)

C. spp.

Horriditriletes sp.

Leiotriletes directus Balme \& Hennelly, 1956 (Figura 3E)

Lundbladispora sp. cf. L. braziliensis (Pant \& Srivastava) Marques-Toigo \& Pons emend. Marques-Toigo \& Picarelli, 1985

L. sp. cf. L. riobonitensis Marques-Toigo \& Picarelli, 1985

Punctatisporites sp. (Figura 3F)

Retusotriletes sp. cf. R. diversiformis (Balme \& Hennelly) Balme \& Playford, 1967 (Figura 3G)

Vallatisporites arcuatus (Marques-Toigo) Archangelsky \& Gamerro, 1979 (Figura 3H)

\section{Granos de polen monosacado}

Accinctisporites ligatus Leschik en Kräusel \& Leschik, 1955 (Figuras 3I-J)

Barakarites sp. cf. B. rotatus (Balme \& Hennelly) Bharadwaj \& Tiwari, 1964 (Figura 3K)

Caheniasaccites elongatus Bose \& Kar, 1966 (Figura 3L)

C. flavatus Bose \& Kar, 1966 (Figura 3M)

C. ovatus Bosé \& Kar emend. Gutiérrez, 1993 (Figura 3N)

C. spp.

Cannanoropollis densus (Lelé) Bose \& Maheshwari, 1968

C. janakii Potonié \& Sah, 1960 (Figura 3U)

C. mehtae (Lele) Bose \& Maheshwari, 1968 (Figura 3P)

Circumplicatipollis plicatus Ottone \& Azcuy, 1988 (Figuras 3Q-R)

Crucisaccites latisulcatus Lele \& Maithy, 1964 (Figura 3S)

Crutaesporites globosus Leschik, 1956 (Figura 3T)

C. sp. A (Figura 3O)

Latusipollenites quadrisaccatus Marques-Toigo, 1974 (Figura 3V)

Mabuitasaccites crucistriatus (Ybert) Playford \& Dino, 2000b (Figura EW)

M. sp. A (Figura 4A)

Potonieisporites lelei Maheshwari, 1967 (Figura 4B)

Stellapollenites sp.

Striomonosaccites cicatricosus Archangelsky \& Gamerro, 1979 (Figura 4C)

S. ovatus Bharadwaj, 1962 (Figura 4F)

Tuberisaccites varius Lele \& Makada, 1972 (Figura 4G)

Granos de polen bisacado

Alisporites australis de Jersey, 1962 (Figura 4D)

A. opii Daugherty, 1941 (Figura 4E)

A. rioclarensis Menéndez, 1976 (Figura 4H)

A. parvus de Jersey, 1962 (Figura 4I)

A. similis Balme, 1957 (Figura 4J)

A. spp.

Chordasporites sp. cf. C. australiensis de Jersey, 1962

Colpisaccites sp. cf. C. granulosus Archangelsky \& Gamerro, 1979 (Figura 4K)

Limitisporites amazoniensis Playford \& Dino, 2000b (Figura 4L) 
L. sp. cf. L. delasaucei Schaarschmidt, 1963 (Figura 4M)

Limitisporites spp.

Platysaccus sp. cf. P. olivae Ottone en Ottone, Alvarez \& Benoit, 1992

P. sp. cf. P. queenslandi de Jersey, 1962

Pteruchipollenites gracilis (Segroves) Foster, 1979 (Figura 4R)

P. spp.

Scheuringipollenites circularis Césari, Archangelsky \& Seoane, 1995

S. sp. cf. S. maximus (Hart) Tiwari, 1973 (Figura 4S)

S. medius (Burjack) Dias-Fabrício, 1981 (Figura 4N)

S. ovatus (Balme \& Hennelly) Foster, 1975

Vestigisporites sp. A (Figura, 4P)

$V$. sp. B (Figura 4Q)

Vitreisporites sp. cf. V. signatus Leschik, 1955 (Figura 4O)

Granos de polen bisacado-teniado

Corisaccites alutas Venkatachala \& Kar, 1966 (Figura 5A)

C. vanus Venkatachala \& Kar, 1966 (Figuras 5D-E)

Hamiapollenites bullaeformis (Samoilovich) Jansonius, 1962 (Figura 4Y)

H. erebi Utting, 1994 (Figura 4U)

H. fusiformis Marques-Toigo emend. Archangelsky \& Gamerro, 1979 (Figura 4T)

H. ruditaeniatus Qu \& Wang, 1986 (Figuras 4V-X)

H. spp.

Illinites unicus Kosanke emend. Jansonius \& Hills, 1976 (Figuras 5B-C)

Lueckisporites sp. cf. L. angoulaensis Jardiné, 1974 (Figura 5H)

L. singhii Balme, 1970 (Figuras 5F-G)

L. singrauliensis Sinha, 1972 (Figura 5I)

L. stenotaeniatus Menéndez, 1976 (Figura 5L)

L. spp.

Lunatisporites ovatus (Goubin) Maheshwari \& Banerjee, 1975 (Figura 5J)

L. sp. cf. L. pellucidus (Goubin) Helby, 1973 (Figura 5K)

L. sp. cf. L. variesectus Archangelsky \& Gamerro, 1979 (Figura 5P)

L. spp.

Protohaploxypinus amplus (Balme \& Hennelly) Hart, 1964 (Figura 5Q)

P. goraiensis (Potonié \& Lele) Hart, 1964 (Figura 5O)

P. microcorpus (Schaarschmidt) Clarke, 1965 (Figura 5M)

P. spp.

Striapollenites sp. A (Figura 5U)

Striatopodocarpites cancellatus (Balme \& Hennelly) Hart, 1965 (Figura 5V)

S. gondwanensis Lakhanpal, Sah \& Dube, 1960 (Figura 5R)

Tornopollenites sp.

Granos de polen plicado

Marsupipollenites striatus (Balme \& Hennelly) Hart, 1965 (Figura 5Y)

Pakhapites fusus (Bose \& Kar) Menéndez, 1971 (Figura 5N)

P. ovatus (Bose \& Kar) García, 1996 (Figura 5T)

Vittatina corrugata Marques-Toigo, 1974 (Figura 5S)

V. costabilis Wilson, 1962 (Figura 5Z)

V. fasciolata (Balme \& Hennelly) Bharadwaj, 1962 (Figura 5AA)

V. subsaccata Samoilovich, 1953 (Figura 5AB)

V. vittifera (Luber \& Waltz) Samoilovich, 1953 (Figura 5X)

V. spp

Weylandites lucifer (Bharadwaj \& Salujha) Foster, 1975 (Figura 5AC)

W. magmus (Bose \& Kar) Backhouse, 1991 (Figura 5W)

Granos de polen polisacado

Polarisaccites bilateralis Ybert \& Marques-Toigo, 1971

Granos de polen sulcado

Cycadopites crassimarginis (de Jersey) de Jersey, 1964 (Figura 5AD)

C. follicularis Wilson \& Webster, 1946 (Figura 5AE)

Algas

Brazilea sp. A Playford \& Dino, 2000b (Figura 5AF)

Fungi indeterminados 